\title{
Organic semiconductor/polymer blend films for organic field-effect transistors
}

\author{
Sergi Riera-Galindo, Francesca Leonardi, Raphael Pfattner, and Marta Mas-Torrent ${ }^{*}$
}

Institut de Ciència de Materials de Barcelona (ICMAB-CSIC) and CIBER-BBN, Campus de la UAB, 08193 Bellaterra, Spain.

E-mail: $\underline{\text { mmas@icmab.es }}$

Keywords: organic field-effect transistor, organic semiconductor blend, solution deposition techniques, device performance

The development of low-cost printed organic electronics entails the processing of the active organic semiconductors (OSCs) by solution-based techniques. However, the preparation of large area uniform and reproducible films based on OSCs inks can be very challenging due to the low viscosity of their solutions that give dewetting problems, the low stability of OSC polymer solutions or the difficulty in achieving appropriate crystal order. To circumvent this, a promising route is the use of blends of OSCs and insulating binding polymers. This approach typically gives rise to films with an enhanced crystallinity and organic field-effect transistors (OFETs) with significantly improved device performance. In this review paper, we overview the recent progress realized in the fabrication of OFETs based on OSC/binding polymer inks highlighting the main morphological and structural features that are playing a major role in determining the final electrical properties and some future perspectives. Undoubtedly, the use of this type of blends results in more reliable and reproducible devices that can be fabricated on large areas and at low cost and, thus, this methodology brings great expectations for the implementation of OSCs in real applications. 


\section{Introduction}

Over the last two decades the field of organic electronics has witnessed a massive increase in the performance of organic field-effect transistors (OFETs) thanks to the intense work devoted, which was motivated by the potential of these devices for low-cost and flexible applications. The active material in OFETs are organic semiconductors (OSCs), which can be either small conjugated molecules or conjugated polymers. ${ }^{[1-5]}$ In order to fabricate cheap devices it is imperative to process the OSCs with solution-based techniques and, hence, to develop what is known as printed electronics. ${ }^{[6-9]}$ Polymeric OSCs tend to be quite soluble in a variety of organic solvents, however, small molecule OSCs commonly suffer from solubility issues. Thus, over the last years novel functionalized OSCs have been reported in which the addition of solubilising side groups to the conjugated and rigid cores has permitted to dramatically improve the material solubility without losing their electronic properties nor disturbing their molecular packing. ${ }^{[10-12]}$ However, another challenge lies on the preparation of large area uniform and reproducible films based on OSCs inks. Especially in small molecule OSCs due to the low viscosity of their solutions, dewetting problems are often encountered leading to non-continuous and inhomogeneous films. To circumvent this, a promising route, which is increasingly been applied, is the use of blends of OSCs and insulating binding polymers. ${ }^{[13-17]}$ Employing blended OSCs has importantly facilitated the solution processability of these materials and, further, has often provided other crucial advantages such as the improvement of the film crystallinity or the device environmental stability. ${ }^{[18]}$ In this review paper, we report the recent advances achieved using thin films of blends of an OSC and an insulating polymer as active materials in OFETs. It should be noticed that although many promising works have also been devoted to the use of blends of a small molecule OSC with a polymer OSC as matrix, this type of materials have not been considered in this overview. The paper is organized by giving first a short introduction on the 
OFET devices and then a summary of the different materials that have been reported followed by the description of the general characteristics of the films in terms of morphology and structure as well as regarding their electrical properties. Finally, the use of blends in electrolyte-gated field effect transistors (EGOFETs) is also introduced as an emerging application area and the future perspectives of these materials are discussed.

\section{OFET configurations and device characteristics}

Briefly, an OFET is a three terminal electronic device where an organic semiconductor is placed on a dielectric and contacted between the source and drain electrodes. The electric field controlled by the gate electrode, which is on the other side of the dielectric, permits to tune the source-drain current. Four possible OFET structures can be defined by the positions of the three electrodes with respect with the organic semiconductor as shown in Figure 1a-d. Further, depending on the charge nature flowing along the organic semiconductor we can classify the transistors as $p$-channel (positive charges) or $n$-channel (negative charges). The first ones achieve their highest conductivity (i.e., “on” state) with negative gate voltages, whereas the latter turn on at positive gate voltages.

OFETs are usually characterised by measuring the source-drain current $\left(I_{D S}\right)$ and either keeping the gate voltage $\left(V_{G S}\right)$ constant and sweeping drain voltage $\left(V_{D S}\right)$, which is referred as output curve (Figure 1e), or by holding $V_{D S}$ constant and sweeping $V_{G S}$, commonly referred to as transfer curves (Figure 1f). The key figures of merit used to quantify the performance of OFETs are: 1) the field effect mobility ( $\mu$ ) (i.e., carrier velocity per unit electric field), 2) the threshold voltage $\left(V_{T H}\right)$, which is the voltage required to induce mobile charges at the organic semiconductor/dielectric interface, 3$)$ the on-off current ratio $\left(I_{O N} / I_{O F F}\right)$ and 4$)$ the subthreshold slope (SS), which is a measure of how fast the device switches from the off state to the on state. 
According to MOSFET theory, there are two operation regimes: linear regime and saturation regime. For a given $V_{G S}$, the drain current first increases linearly with the drain voltage (linear regime), until it saturates reaching a constant value (saturation regime) (Figure 1e). ${ }^{[19]}$

(a)

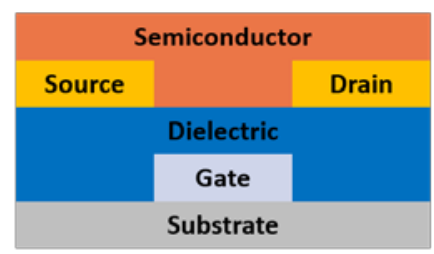

(c)

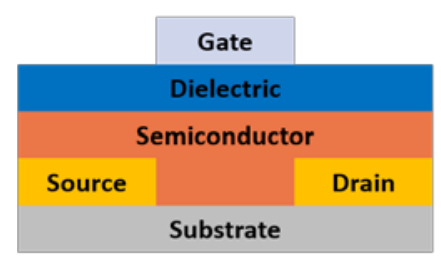

(b)
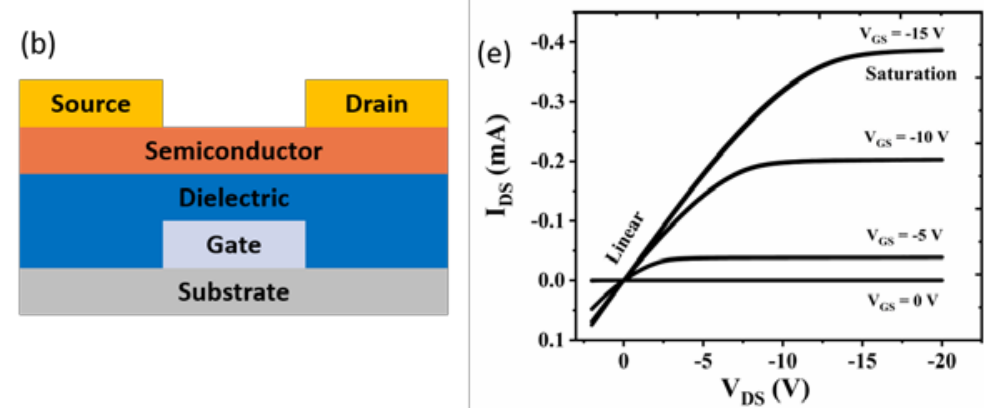

(d)

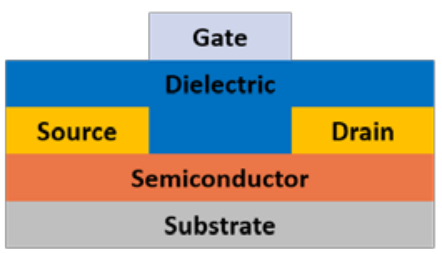

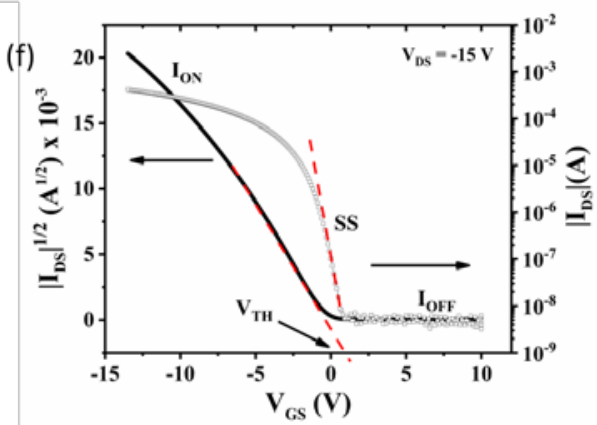

Figure 1. OFET configurations: (a) bottom-gate bottom-contact, (b) bottom-gate top-contact,

(c) top-gate bottom-contact, and (d) top-gate top-contact. Main electrical characteristics: output (e) and transfer (f) of a p-channel OFET.

\section{Overview of different OSC/insulting polymer blends}

\subsection{Blends of small molecule OSCs/insulating polymers}

Small-molecule OSCs tend to form more ordered structures but the preparation of large area uniform films is extremely challenging. These materials were first mainly studied by evaporating them at ultra-high vacuum or by the controlled growth of single crystals. However, these techniques do not merge with low-cost and flexible printed electronics. Thus, the use of polymer binders has been key to reproducibly fabricate crystalline and uniform films boosting their electrical performance and applicability. Table 1 summarises some of the best devices achieved with this type of blends. 
The beneficial effects of polymer binders in small conjugated semiconductors were firstly recognized in a rubrene-based OFET. ${ }^{[20]}$ The deposition of rubrene thin films has been always hampered by its poor film forming capability and its tendency to oxidation. Thus, the studies based on this material were limited to single crystal OFETs, which displayed a very high OFET mobility $>15.4 \mathrm{~cm}^{2} \mathrm{~V}^{-1} \mathrm{~s}^{-1}$. ${ }^{[21]}$ It has been demonstrated that the use of an insulating binder not only improved thin film formation but also offered the possibility to control the OSC crystallization and even the polymorphism of the molecule. As reported by Jo et al. in $2015,{ }^{[22]}$ changing the type of polymer influences the phase separation mechanism during the thin film deposition which in turn has a dramatic effect on the thin film morphology and structure as well as on the electronic properties of the OFET device.

Other acene derivatives have gained popularity due to their impressive electronic performance and easier manipulation with solution processing techniques. For instance, 6,13bis(triisopropylsilylethynyl)pentacene (TIPS-PEN) is a reference high mobility OSC which has been extensively employed in combination with various insulating binders, like PS, ${ }^{[23]}$ poly( $\alpha$-methylstyrene) (PAMS) $)^{[24]}$ and amorphous polycarbonate (APC). ${ }^{[25]}$ Due to the excellent coating capability of TIPS-PEN-based blends, many groups have attempted to fabricate OFETs with several solution shearing and printing techniques, like slot die coating, spray coating or ink-jet printing reaching a field-effect mobility compared to amorphous silicon. ${ }^{[26-28]}$ In a recent report, the thin film deposition of TIPS-PEN:PS by blade coating using different molecular weights PS and different deposition speeds was investigated. By carefully optimizing the coating deposition parameters and formulation, thin films with the typical spherulitic morphology of TIPS-PEN resulted in an outstanding OFET mobility of 8.3 $\mathrm{cm}^{2} \mathrm{~V}^{-1} \mathrm{~s}^{-1} \cdot{ }^{[29]}$

Similarly to TIPS-PEN, blends of 5,11-Bis(triethylsilylethynyl)anthradithiophene (TES-ADT), 2,8-Difluoro-5,11-bis(triethylsilylethynyl)anthradithiophene (diF-TES-ADT) and 2,7-Dioctyl[1]benzothieno[3,2-b][1]benzothiophene (C8-BTBT) have been widely 
employed for OFET fabrication. ${ }^{[13,30-33]}$ Our group developed a unified protocol for the fabrication of OFETs based on OSC-polymer blends by using four well-known organic semiconductors, i.e diF-TES-ADT, TIPS-PEN, C8-BTBT and dithiophenetetrathiafulvalene (DTTTF). ${ }^{[34,35]}$ The deposition of the blends with a solution shearing technique, namely BarAssisted Meniscus Shearing (BAMS), at a high speed of $1 \mathrm{~cm} / \mathrm{s}$ resulted in OFETs displaying state-of-the-art performance with mobilities in the range $0.1-2 \mathrm{~cm}^{2} \mathrm{~V}^{-1} \mathrm{~s}^{-1}$. Remarkably, although at a much lower deposition speed of $250 \mu \mathrm{m} / \mathrm{s}$, excellent device performance with thin films of C8-BTBT:PS blends coated by blade coating have been recently reported, reaching a gate-voltage-independent mobility of up to $12 \mathrm{~cm}^{2} \mathrm{~V}^{-1} \mathrm{~s}^{-1}$. [36]

Dinaphtho[2,3-b:2',3'-f]thieno[3,2-b]thiophene (DNTT) is known as one of the best performing and stable organic semiconductors showing high field-effect mobilities in evaporated thin films $\left(>1.0 \mathrm{~cm}^{2} \mathrm{~V}^{-1} \mathrm{~s}^{-1}\right)^{[37-39]}$ or in single crystals grown by physical vapor transport $\left(8.3 \mathrm{~cm}^{2} \mathrm{~V}^{-1} \mathrm{~s}^{-1}\right) .{ }^{[40,41]}$ However, the poor solubility of DNTT prevents the fabrication of thin films by solution processes. Kimura et al. demonstrated that employing blends of PS and a soluble DNTT precursor, solution-processed thin films could be achieved after an annealing process in which the precursor was converted to DNTT. ${ }^{[42]}$ Subsequently, the devices were optimized by changing the stereoisomer ratio of the DNTT precursors in the blend and the surface treatments to control the position of DNTT layer and the crystal size after the thermal conversion of the precursor to DNTT, reaching an impressive average fieldeffect mobility of $3.35 \mathrm{~cm}^{2} \mathrm{~V}^{-1} \mathrm{~s}^{-1} \cdot{ }^{[43]}$

The addition of a small fraction of PMMA as binding polymer has also been fundamental to improve the crystallisation of the thiophene derivatives $\alpha, \omega$-dihexylquaterthiophene (DH4T) and diketopyrrolopyrrole-sexithiophene (DPP6T) processed by dip-coating. ${ }^{[4]}$ The improved crystallization was attributed to the viscosity gradient at the meniscus during dip-coating imparted by the polymer that facilitates the mass 
transport, and to the fact that the polymer binder solidified at the bottom layer reducing the nucleation barrier height of the small molecule OSCs.

The use of blends has also permitted the preparation of films of promising materials that, however, display poor coating properties or limited solubility. This is the case, for instance, of 3,11-didecyldinaphtho[2,3-d:2',3'-d']benzo[1,2-b:4,5-b']dithiophene $\quad$ (C10DNBDT-NW ${ }^{[45]}$ and meso-diphenyl tetrathia[22]annulene[2,1,2,1] (DPTTA), ${ }^{[46]}$ which only after blending them with insulating matrixes, films showing high performance with mobilities of $>5$ and $1.0 \mathrm{~cm}^{2} \mathrm{~V}^{-1} \mathrm{~s}^{-1}$, respectively, could be fabricated.

Similar to the case of polymer OSCs, most of the blends of small molecule OSCs have been focused on p-type materials. However, we believe that the impact that blending might reveal in $n$-type or ambipolar OSCs could be even more pronounced. Campos et al. explored the influence of the binding polymer in solution-sheared thin films of the $n$-type OSC N,N'bis(n-octyl)-dicyanoperylene-3,4:9,10-bis(dicarboximide) (PDI8CN2) by preparing films based on only the OSC or blended with PS. ${ }^{[47]}$ It was observed that devices prepared including PS exhibited a higher performance and, importantly, a significantly improved bias and environmental stability, which is crucial for the reliability of the devices. The blending route has also been applied in a similar PDI analogue and in a soluble oxidized diketopyrrolopyrrole (DPP) derivative reaching high electron mobilities of 0.16 and $0.5 \mathrm{~cm}^{2} \mathrm{~V}^{-1} \mathrm{~s}^{-1}$, respectively. ${ }^{[48,49]}$

The improved device performance and bias-stress stability was also elucidated in blends of an ambipolar OSC, namely, quinoidal biselenophene (QBS), and the binder polymer poly(2-vinylnaphthalene) $(\mathrm{PVN}) .{ }^{[50]}$ The resulting OFETs exhibited almost four times higher mobility than the neat QBS semiconductor, giving hole/electron mobilities of $0.08 / 0.02 \mathrm{~cm}^{2} \mathrm{~V}^{-}$ ${ }^{1} \mathrm{~s}^{-1}$. 
Table 1. Some of the best performance OFETs based on blends of small molecule OSCs and insulating binders.

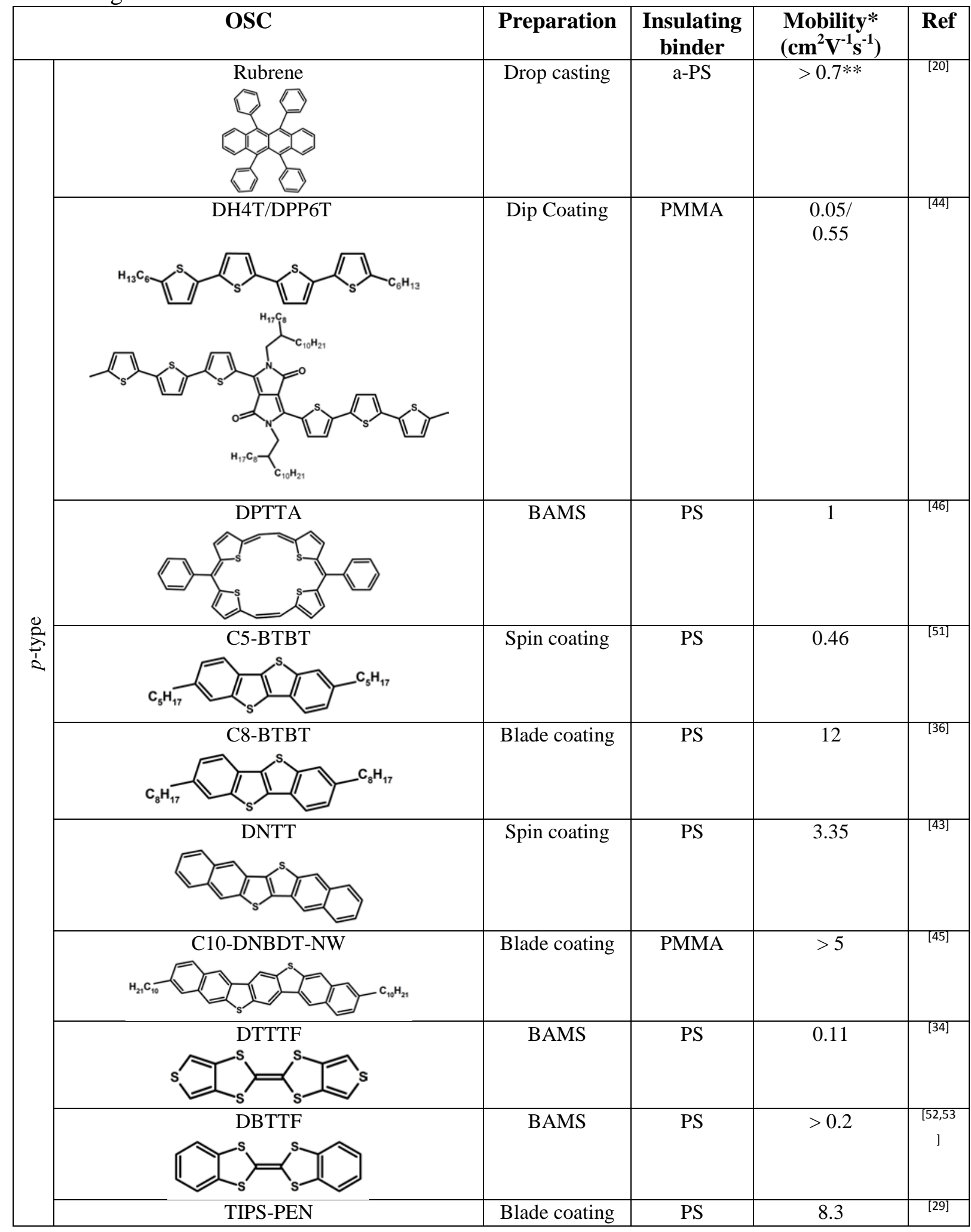




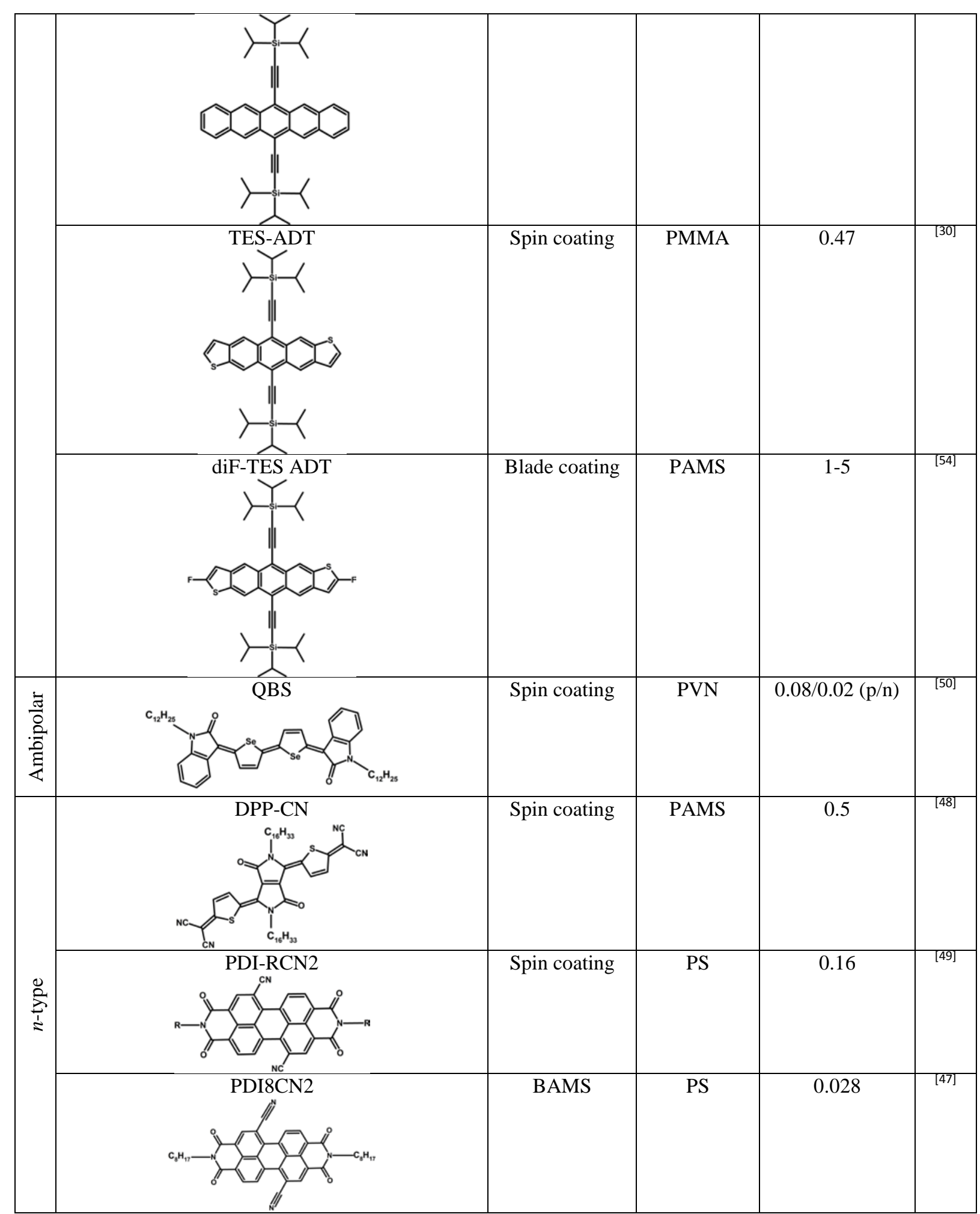

*Values declared by authors and extracted in the saturation regime. If maximum and average mobility are reported, only the average one is included.

**A vitrifying agent is also present. 


\subsection{Blends of polymer OSCs/insulating polymers}

Although the use of binding polymers has a more significant impact on improving the wettability of small molecule OSCs, it can also offer clear benefits when they are blended with polymeric OSCs. Table 2 displays some of the best mobility values reported for different blended polymer OSCs along with their molecular structure.

One of the most studied polymer OSCs is poly(3-hexylthiophene-2,5-diyl) (P3HT), which in the late 90s' reached one of the highest OFET mobility of $0.1-0.2 \mathrm{~cm}^{2} \mathrm{~V}^{-1} \mathrm{~s}^{-1}{ }^{[55]}$ It was found that the deposition technique was fundamental for ensuring a proper selforganization of the well-known lamellar structure of this polymer, ${ }^{[56]}$ which is required for achieving a high mobility P3HT-based OFET. Another drawback of this material is its low air-stability typically giving rise to devices with high positive threshold voltage $\left(V_{t h}\right)$. Thin films of P3HT-based blends have been intensively studied in combination with different insulating binders like polyethylene (PE) or high-density polyethylene (HDPE), ${ }^{[57]}$ polystyrene (PS) (amorphous and isotactic), ${ }^{[57-59]}$ poly (methyl methacrylate) (PMMA), ${ }^{[60]}$ poly(lactic acid) (PLA), ${ }^{[61]}$ polyacrylonitrile (PAN) ${ }^{[62]}$ and poly(vinyl cinnamate) (PVCn). ${ }^{[63]}$ In all these cases, the vertical phase separation occurring during thin film deposition helped the crystallization of P3HT and, importantly, the environmental device stability was strongly enhanced. Further, the amount of the active material could be strongly reduced, since the best performances were achieved by using only a typical proportion in the range $2-10 \%$ of the OSC. Mobilities reaching and even surpassing the ones previously reported by the bare polymer were normally found.

Although P3HT is still considered a benchmark polymer OSC, the impressive progress in chemical synthesis of novel semiconducting polymers has resulted in a vast library of highmobility polymers. ${ }^{[17]}$ Most of them are actually thiophene-based polymers and copolymers. Despite the impressive mobility values reported, ${ }^{[64]}$ these polymers commonly suffer from the same drawbacks as P3HT: a high crystallinity or operational stability are often difficult to 
achieve. Further, the best performing materials tend to be high molecular weight polymers, which although they can be dissolved with hot solvents, their solutions are often not very stable due to gelling or precipitation. The use of blend inks has revealed to be an appealing route to solve all these problems since, in addition to facilitate the crystallization and improve the stability, a lower loading of the polymer semiconductor is required yielding a more stable solution. Lei and co-workers ${ }^{[62]}$ demonstrated a two-orders magnitude enhancement of the OFET electrical characteristics in spin coated films of the semiconductor diketopyrrolopyrrole-dithienylthieno[3,2-b]thiophene $(\mathrm{P}(\mathrm{I})$ ) when it was mixed with PAN, with a reported charge carrier mobility as high as $11.43 \mathrm{~cm}^{2} \mathrm{~V}^{-1} \mathrm{~s}^{-1}$. Remarkably, on par mobility values have also been achieved with polymer OSC blends employing techniques such as solution shearing ${ }^{[65]}$ and inkjet printing, ${ }^{[62]}$ paving the way to more appealing and industrial scalable approaches. It should be highlighted that the blending strategy has been mainly applied in $p$-type polymer semiconductors, but an example of an ambipolar polymer OSC has already been reported, despite the fact that the $n$-type characteristics were only preserved storing the devices in a $\mathrm{N}_{2}$ atmosphere. ${ }^{[66]}$

Table 2. Some of the best performance OFETs based on blends of polymeric OSCs and insulating binders.

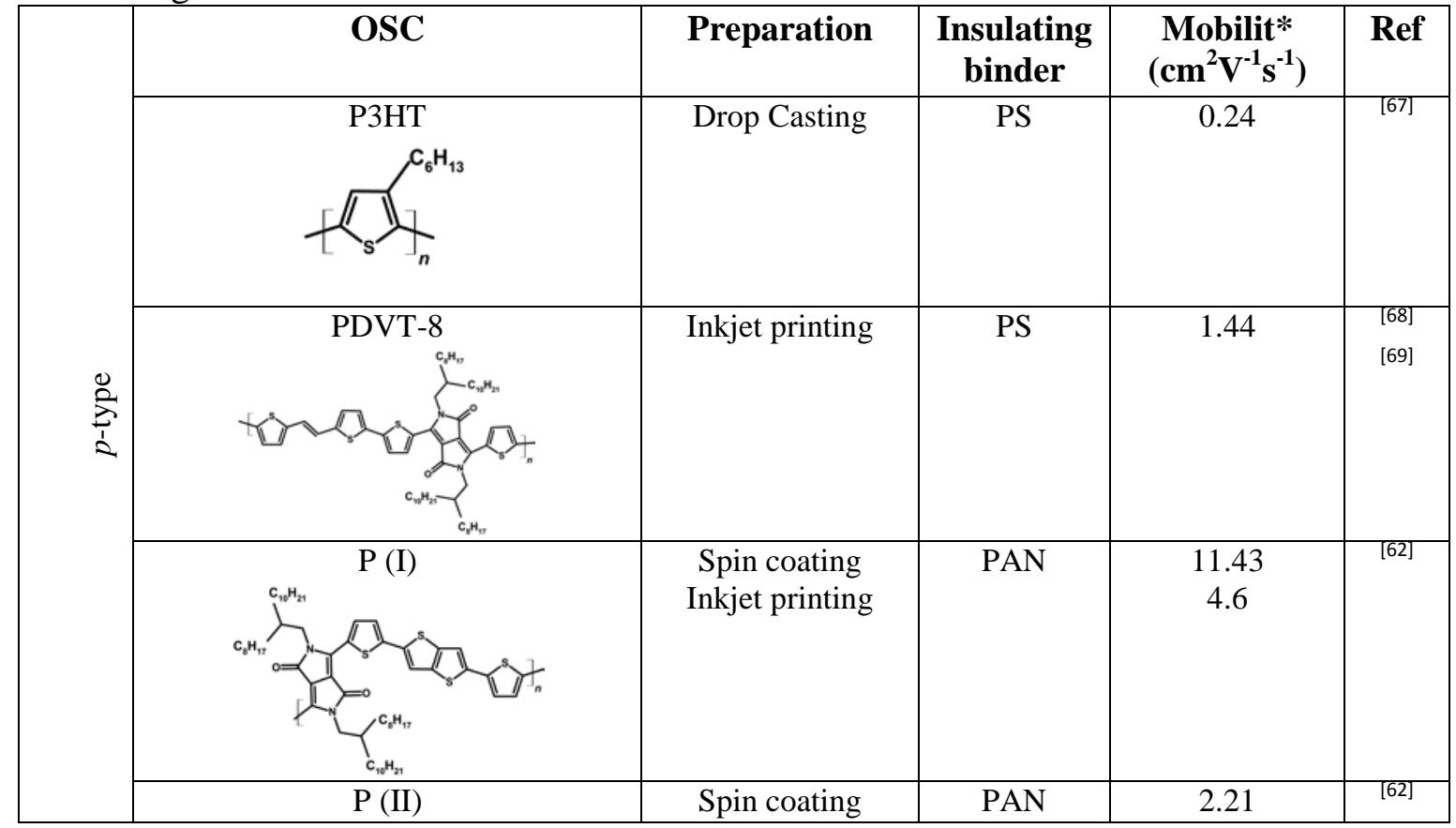




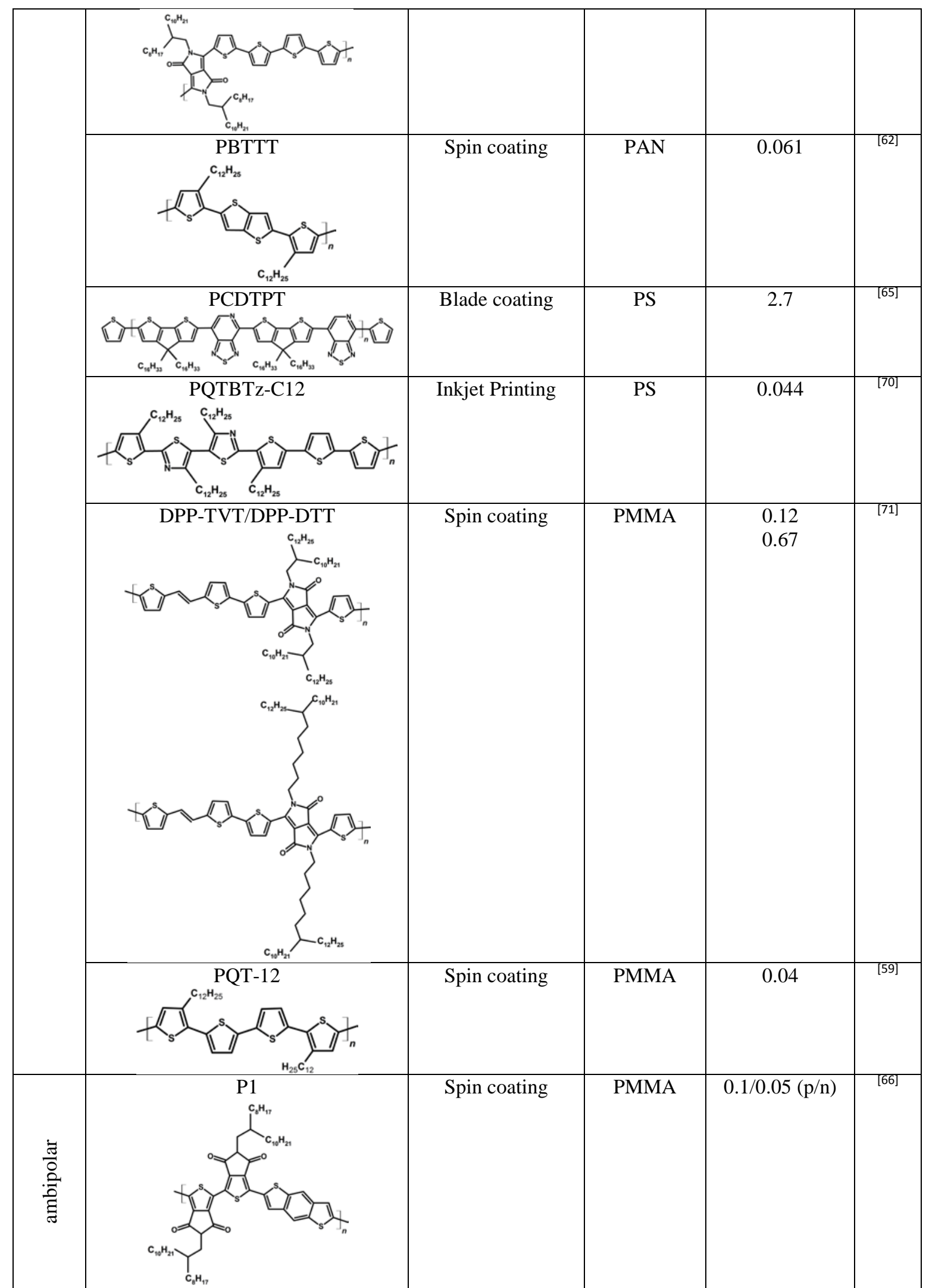

*Values declared by authors and extracted in the saturation regime. If maximum and average mobility are reported, only the average one is included. 


\section{Morphology and structural thin film characterization}

Using OSC inks with two components, greatly increases the difficulty in controlling and understanding the final thin films microstructures that will be formed. ${ }^{[13,14,72]}$ A large number of factors should be taken into account ranging from the solvent boiling point, chemical properties of the materials, processing conditions, type of substrate, etc., which makes it extremely complex to predict the final thin films characteristics. However, all the work that has been carried out in the last few years can provide very useful guidelines related to the chemical, kinetic and thermodynamic factors that are playing a major role.

\subsection{Thin films morphology}

To obtain charge transport from one electrode (source) to the second electrode (drain) at least one fully percolative pathway, corresponding to the lowest dimensionality (i.e. 1D effective electrical conduction), is needed. With increasing dimensionality of effective charge pathway, charge carrier mobility and device performance increase ${ }^{[73-75]}$ Horizontal or lateral phase separation is easy to identify with techniques such as Polarised Optical Microscopy (POM), Atomic Force Microscopy (AFM) and Scanning Electron Microscopy (SEM), among others.

The lateral phase separation has been studied in different polymer OSCs blends. Wirecoating of P3HT and HDPE over a range of blend compositions reveal strong dependence of the initial composition on the final thin film microstructure. ${ }^{[76]}$ In particular, employing 50:50 (OSC:insulator blend) laterally segregated structures with crystalline P3HT-rich along with HDPE domains were found. A similar phenomenon was also found for diketopyrrolopyrrole (DPP)-based semiconducting polymer:PMMA blends, where domain size and related device performance could be efficiently controlled by side chain engineering of the semiconductor. ${ }^{[71]}$ 
The growth mechanism of spin coated films of blends of diF-TES-ADT and PS have been careful investigated by scanning probe microscopies. ${ }^{[77]}$ The authors postulated a model revealing four morphologically distinct crystalline regimes: nucleation centres at the electrodes, crystalline domains with petal-like structures, needles and 3D crystals (Figure 2). These regimes are imposed by five major kinetic parameters: rate of heterogeneous nucleation on the electrodes, rate of crystal growth, rate of solvent evaporation, rate of OSC diffusion in solvent and rate of homo-nucleation in the channel region. Thus, OFET characteristics ranged from poor to high performance and reproducibility depending on the disruption of the crystallization process. The authors envisage that tailoring all these kinetic factors would allow for a control of large length scale crystallites in the device channel.

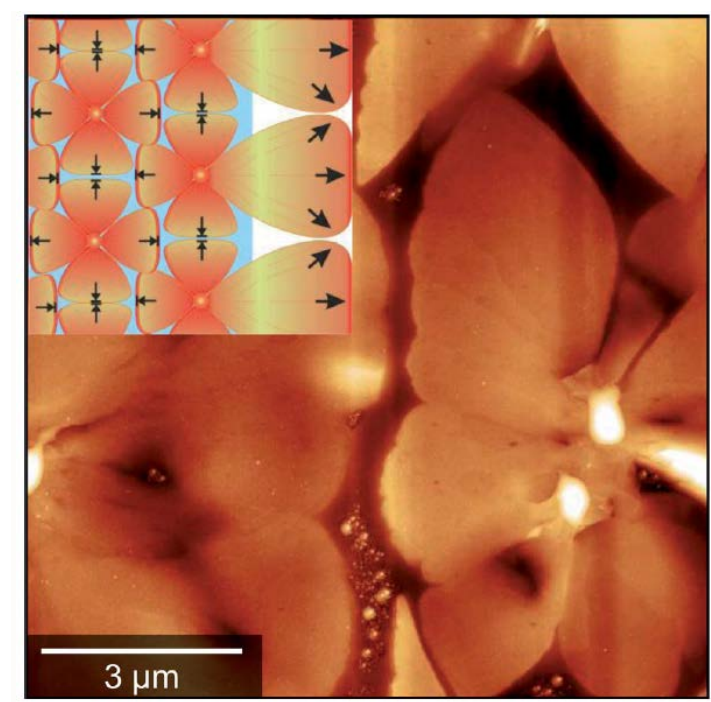

Figure 2. AFM image collected from a top of a PFBT-treated silver electrode where a film of diF-TES-ADT:PS has been spin-coated, showing petal-like grains fanning out from central nucleation centres. Inset: schematic representation of the grain growth process showing the extension of grains from the electrode (blue) into the bulk (white). Reproduced under the terms of the Creative Commons Attribution 3.0 International License. ${ }^{[77]}$ Copyright 2014, the authors, published by The Royal Society of Chemistry. 
The nucleation of the small molecule OSCs diF-TES-ADT and TIPS-PEN had been previously shown that can be enhanced by modification of the gold source-drain electrodes with a self-assembled monolayer of pentafluorobenzenethiol (PFBT). ${ }^{[54,78,79]}$ Such SAMs were found to be detrimental for the preparation of thin films of blends of these molecules deposited by blade coating in the speed range $0.5-2 \mathrm{~mm} / \mathrm{s}$ since they were disrupting the formation of large crystalline domains. ${ }^{[54]}$ However, in blended films prepared by bar-assisted meniscus shearing at $10 \mathrm{~mm} / \mathrm{s}$, devices based on PFBT-modified electrodes revealed an improved performance. $^{[34]}$

The nature of the polymer binder also determines the crystallisation of the OSC. As it will be discussed later on, the polymer can strongly affect the stratification in thin films as well as the extend of crystalline order due to competing effects such as confinement entropy, interaction energy with the surface and solidification kinetics. ${ }^{[80,81]}$ In addition to the type of polymer employed, the molecular weight $\left(M_{w}\right)$ of a polymer might have a remarkable effect on the thin film and, hence, the electrical device characteristics. ${ }^{[29]}$ The $M_{w}$ determines the viscosity, solubility, and miscibility of the materials. Niazi et al. ${ }^{[33]}$ observed that blade sheared films of blends of diF-TES-ADT:PS revealed higher mobilities when increasing the $M_{w}$ of PS, which was mainly accounted for the higher viscosity of the solution that gave rise to crack-free and smoother crystalline domains (Figure 3). 


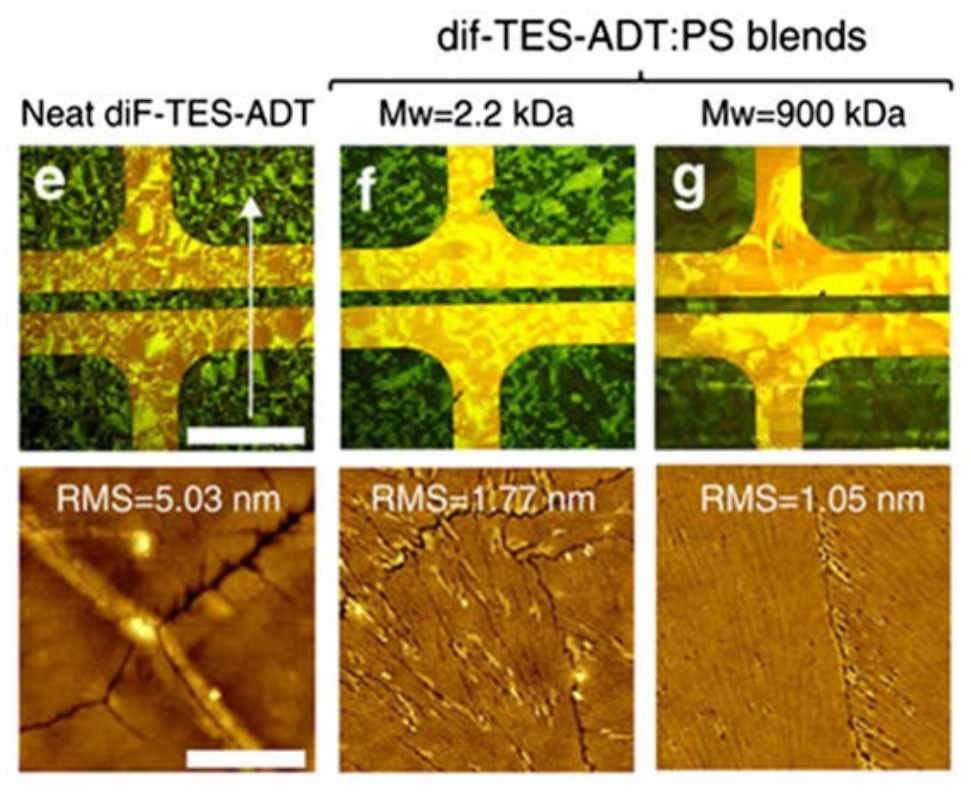

Figure 3. Polarized optical micrographs and atomic force microscopy (AFM) images of (e) neat diF-TES-ADT, (f) low Mw blend (PS $2.2 \mathrm{kDa}$ ), and (g) high Mw blend (PS $900 \mathrm{kDa}$ ). The white arrow shows the direction of blade coating. Scale bars: $250 \mu \mathrm{m}$ [in polarized optical microscopy (POM) images] and $4 \mu \mathrm{m}$ (in AFM images). The thin films were deposited by blade coating. Reproduced under the terms of the Creative Commons Attribution 4.0 International License. ${ }^{[33]}$ Copyright 2015, the authors, published by Springer Nature.

Another parameter that can affect the thin film microstructures is the choice of solvent, which determines the solubility of the materials and the evaporation speed. ${ }^{[33,82]}$ An elegant route called after dual solvent crystallisation has showed to be efficient to control the thin film morphology of small molecule OSC-based blends, and, concomitantly the device performance. It consists in selecting a better solvent for the small molecule and a second solvent which solubilises better the polymer. Hence, in films of TIPS-PEN:PS a morphology transition from needle-shaped (one solvent) to platelet-shaped (dual solvent) was observed, which was ascribed to an improvement of the crystallisation kinetics during phase separation. The use of two solvents has also been applied for ink the jet printing of blends of a polythiophene 
derivative with PS, observing that morphologies with more crystalline domains were reached when using a high boiling point co-solvent. ${ }^{[68]}$

The thin films morphologies are strongly dependant on the processing conditions. A recent study reports the evolution of TIPS-PEN crystallites when varying the spin-coating time (Figure 4). ${ }^{[83,84]}$ When the spin coating time was in the range $3-5 \mathrm{~s}$, one dimensional (1D) TIPS-PEN structures were observed due to an excess of residual solvent that induced a drying-mediated convective flow in a droplet. When the spin-coating time was increased to more than $50 \mathrm{~s}$, the crystal growth mode changed giving two dimensional (2D) spherulites. Larger times were causing a decrease in the crystal size.
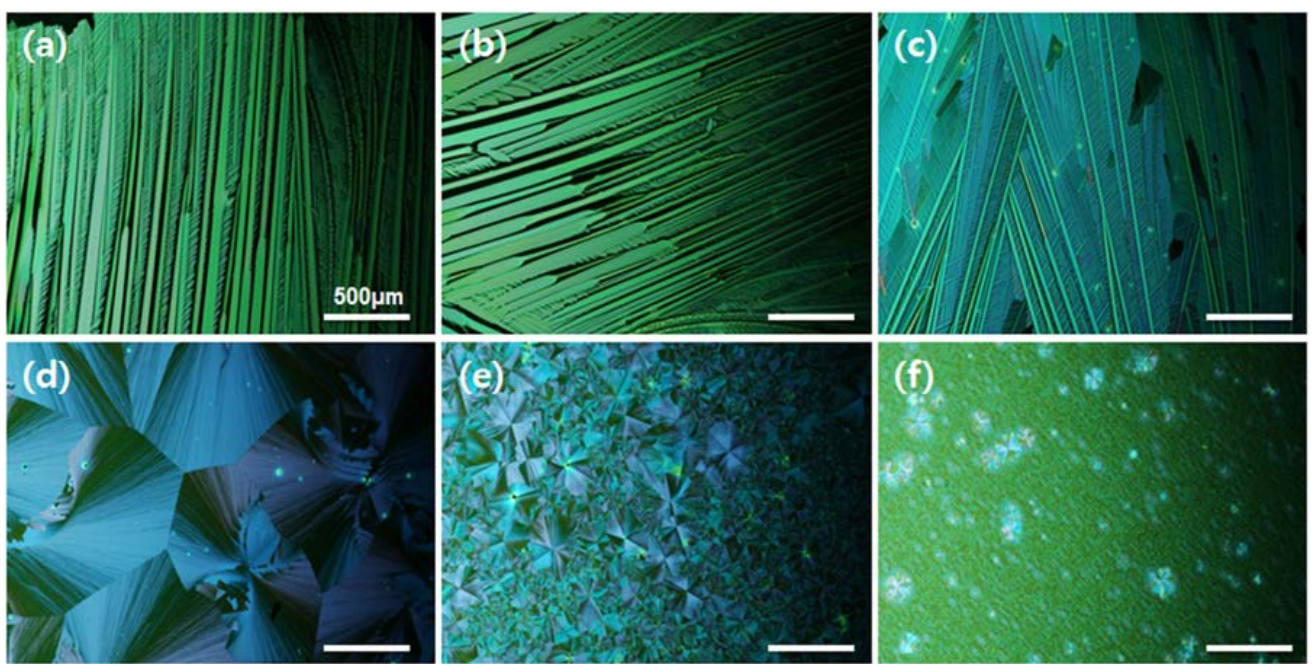

Figure 4. Polarized optical microscopy images of TIPS-pentacene:PS films spun-cast from blend solutions in 1,2-dichlorobenzene over different spin coating times: (a) $3 \mathrm{~s}$, (b) $5 \mathrm{~s}$, (c) $20 \mathrm{~s}$, (d) $50 \mathrm{~s}$, (e) $80 \mathrm{~s}$, and (f) $120 \mathrm{~s}$. Reproduced under the terms of the Creative Commons Attribution 4.0 International License. ${ }^{[84]}$ Copyright 2019, the authors, published by Springer Nature.

In single crystalline materials charge transport depends heavily on the direction of the charge path through the crystal (i.e. energy landscape). ${ }^{[85]}$ In fact, the same crystal typically leads to very different mobility values, depending on the crystal plane and the direction of 
charge transport. ${ }^{[21]}$ For solution processed blends the casting direction may give rise to an additional degree of electrical anisotropy originating from the thin film morphology. The deposition speed in directional coatings techniques has a strong impact on the film anisotropy. Accordingly, slow deposition techniques tend to give long ribbon-like microstructures, whereas fast deposition processes lead to more isotropic $2 \mathrm{D}$ crystallites. ${ }^{[16,29,44,52]}$ Employing the vertical flow method TIPS-PEN crystals were found to grow preferentially normal to the solution flow resulting in crystalline stripes on top of isotactic PMMA with amorphous TIPSPEN regions in between. Device performance strongly depended on the orientation of the transistor channel with the growing direction and exhibited an anisotropy-ratio for saturation mobility of up to $10 .{ }^{[86]}$ In blade coated films deposited at $250 \mu \mathrm{m} / \mathrm{s}$ the thin film anisotropy was explored in films from the neat C8-BTBT OSC and with different C8-BTBT:PS ratios (Figure 5). ${ }^{[36]}$ The extracted average mobility values measured both parallel $\left(0^{\circ}\right)$ and perpendicular to the shearing direction $\left(90^{\circ}\right)$ revealed a pronounced anisotropy for neat devices and those prepared from 1:1 blend solutions with very low mobility perpendicular to the blade movement, which was consistent with the low presence of connected ribbons. On the contrary, the high polymer fraction films such as 1:2 blends exhibited comparable mobility in both directions.

Further, Ford et al. demonstrated that blade-coating onto nanogrooved substrates, realised by scratching with a diamond lapping film, can promote the alignment of a polymer OSC blended with PS. This alignment caused a $\mu$ anisotropy of $\sim$ 8-14 in favour of the nanogroove direction. ${ }^{[65]}$ 


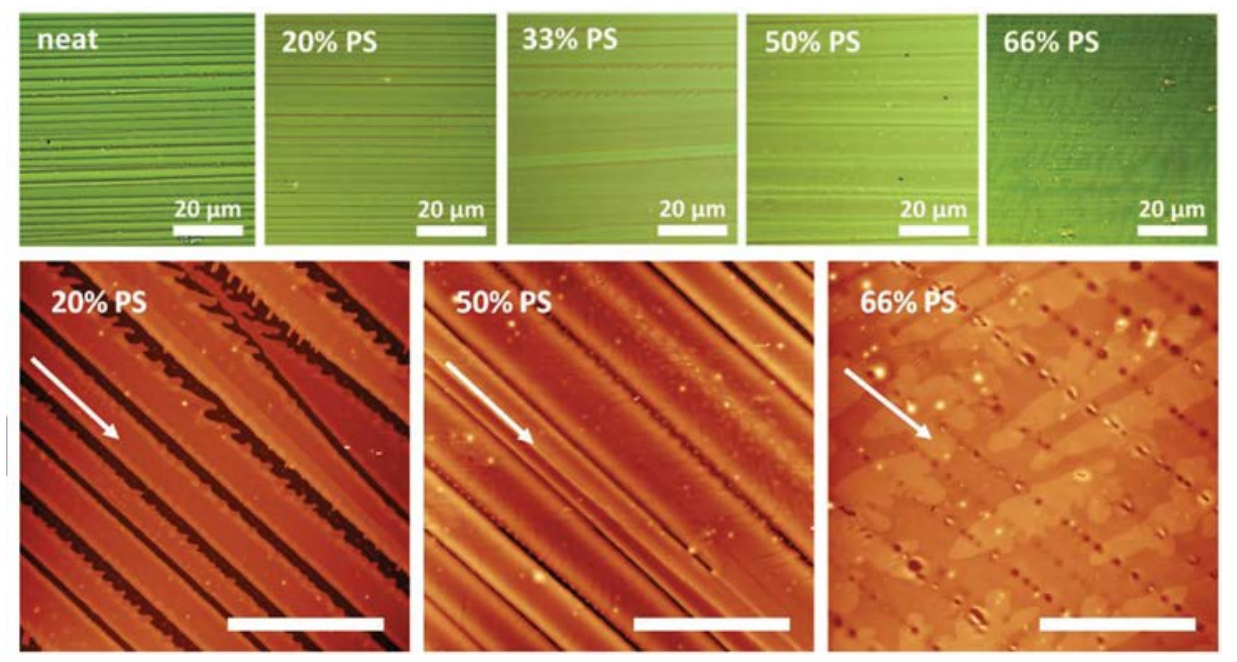

Figure 5. Top) Microscopic images of films prepared from C8-BTBT blends with various ratios. Bottom) AFM images showing magnified views of films prepared with three different fractions of PS; arrows indicate shearing direction; scale bar: $20 \mu \mathrm{m}$. Reproduced with permission. ${ }^{[36]}$ Copyright 2018, Wiley-VCH.

\subsection{Vertical phase separation}

As previously mentioned, during the deposition of OSCs with binding polymers typically a vertical phase separation takes place which is affected both by molecular and processing parameters. It is well known that charge transport in OFETs occur in the first few mono-layers of the active material adjacent to the gate dielectric. ${ }^{[87]}$ Thus, it is essential to ensure that the dielectric/OSC interface is homogenous, continuous and well defined. Partial or incomplete phase separation at this interface can lead to charge trapping sites and must be avoided to guarantee high device performance and bias stability. Unfortunately, the characterization of the vertical phase separation is not straightforward. Typical techniques employed to gain such information are the characterization of the depth profiles (realized with sputtering inert gas ions) by X-ray photoelectron spectroscopy (XPS) and time-of-flight secondary ion mass spectrometry (ToF-SIMS), neutron reflectivity experiments, angle resolved X-ray spectroscopy or energy filtered transmission electron microscopy (EFTEM). 
When a solution of an OSC and a polymer binder is deposited on a substrate the formation or bilayer or even trilayer structures is commonly observed. For instance, it has been reported that in blends of TIPS-PEN:PS deposited by spin coating or bar-assisted meniscus shearing bi-layer structures where TIPS-PEN crystallised on a PS layer were formed. ${ }^{[34,88]}$ However, when the same active material was blended with PAMS and spin coated, tri-layer films where obtained since the TIPS-PEN migrated towards the bottom and top interfaces. ${ }^{[1,89,90]}$ Clearly, the nature of the polymer binder has an important influence on the vertical phase separation. Madec et al. found that in drop-casted TIPS-PEN blend films, amorphous binders typically lead to small or negligible stratification due to larger molecule-polymer interactions in the solidified film. Quiet in contrary, when crystalline binders (e.g. isotactic PS) which crystallize slower than the OSC are used, stratification towards both interfaces appear. ${ }^{[91]}$ The vertical phase separation was also explored in spin coated films of diF-TES-ADT employing as binding polymers PAMS, PMMA and syndiotactic PS (i.e., s-PS). ${ }^{[80]}$ The latter was chosen to test a crystalline binding polymer able to crystallise faster than the OSC. Neutron reflectivity can provide nanometer resolution of depth profiles in composites originating from the appearance of low and high frequency Kiessig fringes. However, in the presence of broad inter-facial widths, the fringes become damped and modelling of the diffuse interfaces is necessary. Fresnel-normalized reflectivity $\left(\mathrm{R} \times \mathrm{Q}^{4}\right)$ indicated that in the case of PAMS again a tri-layer structure was formed, with PMMA the OSC was primary concentrated on the bottom part of the film and with s-PS a solution-like uniform dispersion with no clear phase separation was observed (Figure 6). The devices based on PAMS exhibited the best performance. 
(a)

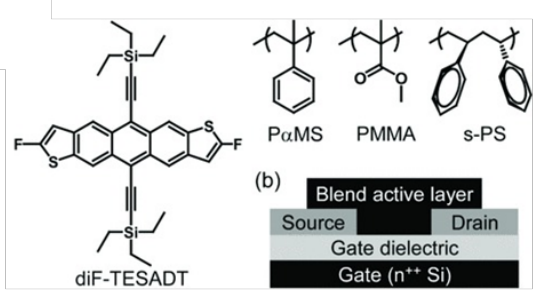

(c)

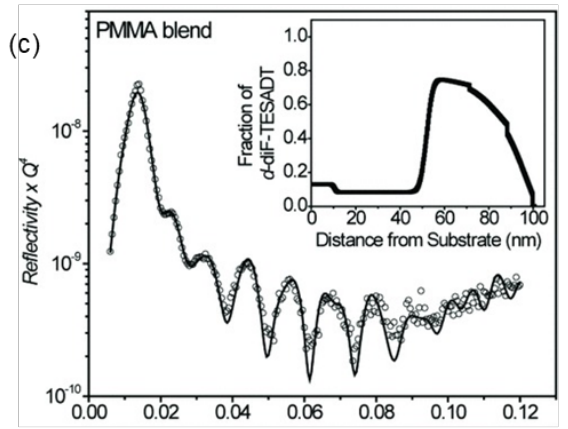

(b)

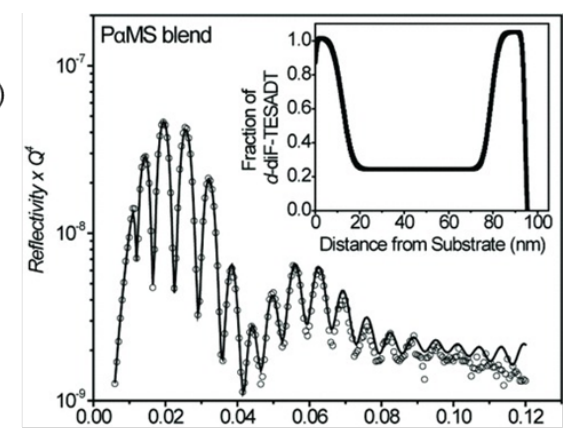

(d)

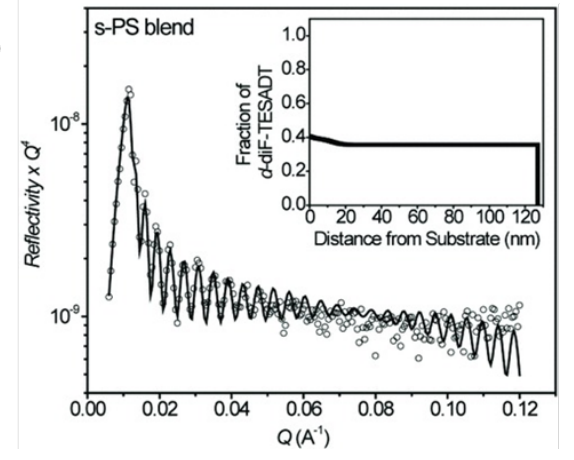

Figure 6. (a) Chemical structures of diF-TES-ADT and various binder polymers employed including schematic structure of bottom gate/bottom contact OFET device. Vertical segregation profiles measured by Neutron Reflectivity (NR) measured in blends of polymer diF-TES-ADT and (b) PAMS (c) PMMA and (d) s-PS. Insets show the volume fraction depth profile of diF-TES-ADT in each blend. Reproduced with permission. ${ }^{[80]}$ Copyright 2013, Wiley-VCH.

The hydrophobicity of the polymer and the OSC as well as the substrate should also be taken into account as they determine the molecular or molecule-substrate interactions. ${ }^{[80,81]}$ For instance, by controlling the surface energy the separation of the OSC and the binder polymer can be modulated. In films of a polythiophene OSC and PMMA deposited by spin coating on a $\mathrm{SiO}_{2}$ dielectric functionalised with a hydrophobic $\mathrm{SAM}$, the polymer was found to be mainly on the bottom part and the PMMA was covering it acting as encapsulating layer and providing an enhanced environmental device stability. ${ }^{[59]}$ In sharp contrast, the opposite stratification was observed when hydrophilic substrates were used. ${ }^{[92]}$ Similarly, modification 
of the gold electrodes can also play a role. In films of TIPS-PEN:PAMS deposited by bladecoating on $\mathrm{SiO}_{2}$ substrates containing either gold electrodes or PFBT-modified gold electrodes, it was shown that bi-layer structures were formed on the substrates with untreated electrodes but, instead, in substrates with PFBT/Au electrodes a clear tri-layer stratification was observed (Figure 7). ${ }^{[54]}$ Additionally, OSCs which are more hydrophilic can also show a preference to interact with hydrophilic substrates, as found in blends of the ambipolar OSC QBS. ${ }^{[93]}$

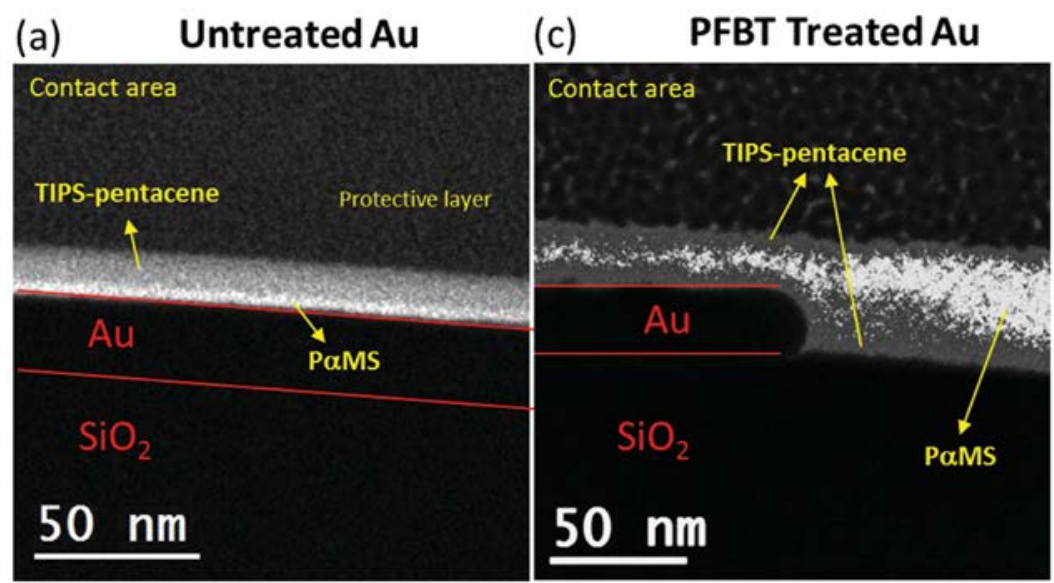

(b)

(d)
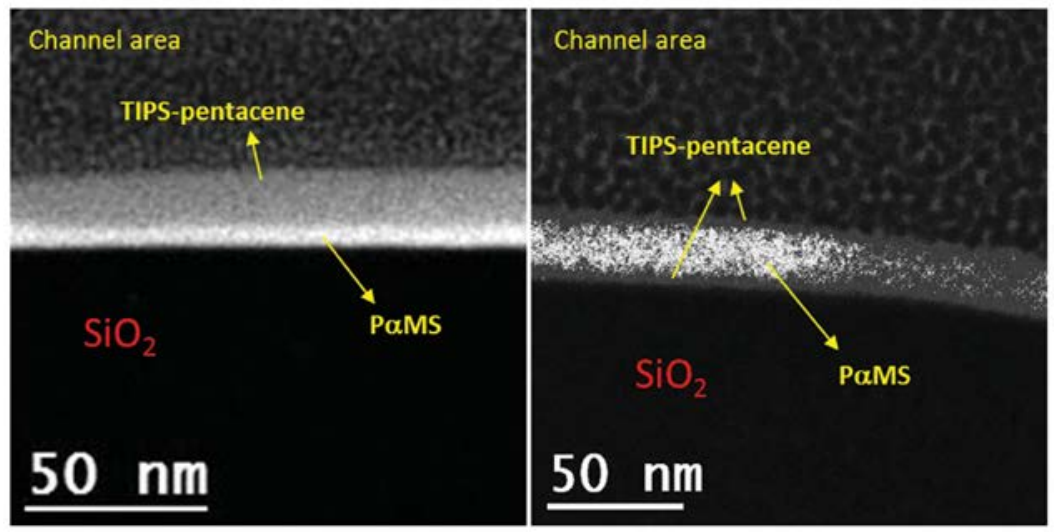

Figure 7. Cross-sectional EFTEM showing the vertical phase separation of TIPS-pentacene: PAMS blend films blade-coated on substrates with a,b) untreated Au contacts (plasmacleaned) and c,d) Au contacts treated with PFBT. Reproduced with permission. ${ }^{[54]}$ Copyright 2015, Wiley-VCH. 
As previously mentioned for the lateral phase separation, the solvent is also an active component. Generally, it has been demonstrated that higher boiling point solvents give rise to more segregated structures due to the decrease in the evaporation rate. ${ }^{[24,94]}$

The use of post-treatments can also alter the material distribution within the film. In films of TIPS-PEN with high molecular weight PAMS, post thermal annealing of the devices did not affect the film structure. In contrast, the same treatment carried out in films with low molecular weight PAMS promoted the migration of the OSC towards the top surface. ${ }^{[90]}$ However, the thermal treatment of a blend film of a DNTT precursor performed to convert the material to the parent OSC, led to the segregation of the DNTT molecules to the interface with the substrate, which probably mediated the crystallisation of the active material. ${ }^{[42]}$ Alternatively, solvent vapour annealing has also been shown to allow for the migration of OSC molecules towards the air-interface to minimise the total surface energy of the film and also to induce the OSC crystallisation. ${ }^{[30]}$

Very often it is stated that OFETs based on OSC blends show better air-stability. ${ }^{[47,53]}$ Recently we have reported that in OSC:binding polymer blends an ultrathin skin-like layer can be formed, which helps to stabilise the devices against diffusion of water and/or oxygen. In bar-coated films of C8-BTBT:PS characterized by friction force microscopy (FFM) it was demonstrated that the blend film was composed by few molecular layers of C8-BTBT with crystalline order, sandwiched between a PS-rich layer at the bottom (a few nm thick) and a PS-rich skin layer on top $(\sim 1 \mathrm{~nm}) .{ }^{[95]}$ Frictional force occurring between the AFM-tip and the sample is converted into cantilever's torsion, permitting to simultaneously extract frictional and topographic images. Figure 8 shows the phase separation from the lateral force signal that allows relating the friction contrast with the material composition, that is, high friction for crystalline C8-BTBT and low friction for PS areas, respectively. 


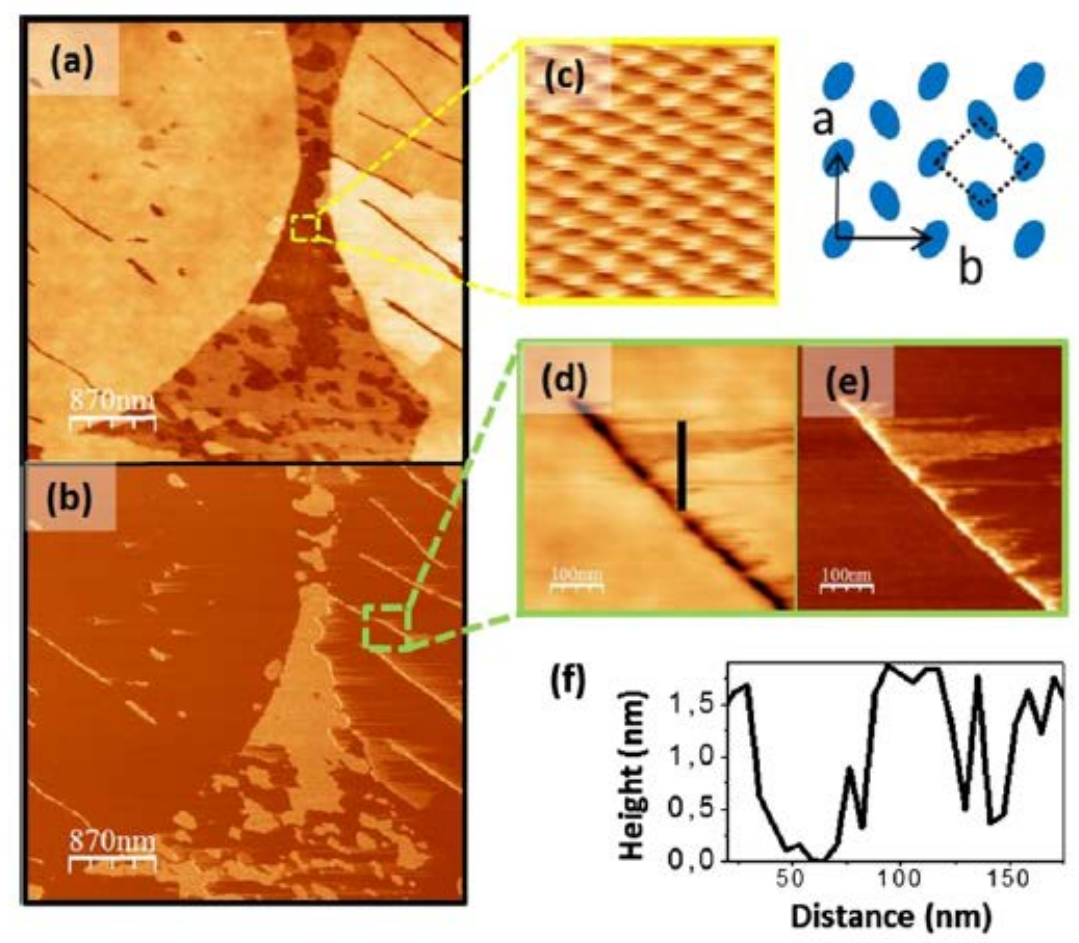

Figure 8. Composition of C8-BTBT:PS blend measured by friction force microscopy (FFM).

(a) Topographic and (b) lateral force images obtained within the channel of the 1:2 blend. (c) Magnified molecular resolution image of C8-BTBT and top-view model of the in-plane herringbone unit cell Blue ellipsoids represent the methylene groups. (d) Topographic and (e) lateral force images obtained at the indicated area. (f) Height profile along the black line traced in (d). Reproduced with permission. ${ }^{[95]}$ Copyright 2018, American Chemical Society.

\subsection{Polymorphism}

Polymorphism is commonly encountered in small molecule OSCs due to the presence of weak van der Waals intermolecular interactions that makes these materials prone to structural modifications. ${ }^{[96,97]}$ It is widely known that the device performance is strongly dependant on the polymorph and, thus, it is imperative to gain control on the polymorph formed or the polymorph purity in an OSC film. The modification of any experimental deposition condition (i.e., temperature, speed, solvent, etc.) is bound to promote structural modifications to the material, and the use of polymer binders is not an exception. ${ }^{[16]}$ 
In films of TIPS-PEN films it was previously found that the fraction of the high mobility polymorph (type IIb) relative to the low mobility polymorph (equilibrium phase polymorph, type I) strongly depends on the film thickness. ${ }^{[98]}$ The same trend has recently been confirmed in blended TIPS-PEN films. ${ }^{[29]}$ Blade-coated films based on high $M_{\mathrm{w}}$ (e.g., $200 \mathrm{kDa}$ ) PS showed a very clear signature of the high-mobility polymorph II when sheared at $2 \mathrm{~mm} \mathrm{~s}^{-1}$ (thickness $<15 \mathrm{~nm}$ ), whereas pristine samples sheared at the same speed (thickness $>20 \mathrm{~nm}$ ) still showed predominantly polymorph type I. As a consequence, a vast improvement in the OFET mobility was achieved in the blended samples.

As mentioned, it has been demonstrated that the type of polymer used in rubrene-based inks can alter the polymorph formed. ${ }^{[22]}$ When PS or poly(4-vinylphenol) (P4VP) is employed, rubrene crystallizes in an orthorhombic structure, while rubrene PMMA blends are more disordered and contain a significant fraction of triclinic phase. Remarkably, only the orthorhombic phase exhibited field-effect response.

Worth mentioning is that not only the nature of the polymer or its $M_{w}$ but also the ratio OSC:binding polymer can affect the formation of different polymorphs, as reported for blends of dibenzotetrathiafulvalene (DBTTF) and PS. ${ }^{[52]}$ Thin films deposited by bar-coating from blends with more than 50 wt \% of DBTTF content showed oriented crystals along the casting direction, but they corresponded to a mixture of crystals from the $\alpha$ (thermodynamic) and $\gamma$ (kinetic) polymorphs. On the other hand, the thin films deposited from 1:1 blends or blends containing less than 50 wt \% of DBTTF were more homogeneous with an isotropic plate-like crystalline domain and belonged to the $\gamma$-phase. Importantly to notice is the presence of mixtures of polymorphs has a detrimental effect on the electrical performance of the devices. 


\section{Electrical characteristics of thin films of blends of OSCs/ insulating binding polymers}

The use of binding polymers not only is helpful to facilitate the materials solution processability but also can give rise to devices with better electrical properties regarding reproducibility, improved OSC/dielectric interface, operational and shelf stability and mechanical properties.

\subsection{OSC/dielectric interface}

Although organic printed electronics is demanding to move to plastic substrates, still nowadays oxide (i.e., $\mathrm{SiO}_{2}$ ) dielectrics are widely used. However, it is well-recognized that the hydroxyl groups present in such dielectrics act as charge traps in detrimental to the device mobility. ${ }^{[99]}$ To avoid this, the passivation of the dielectric with hydrophobic self-assembled monolayers (SAMs) or with buffer polymeric layers has been pursued. ${ }^{[100]}$ When an OSC is blended with an insulating polymer, by selecting the appropriate conditions (i.e., processing technique, coating speed, molecular weight of binding polymer, etc.), vertically segregated films where the OSC is resting on top of a binding polymer layer can be formed, as explained in the previous section. Remarkably, it has been demonstrated that in films showing such structure the binding polymer acts as a passivating layer and greatly reduces the number of interfacial traps at the OSC/dielectric interface, resulting in OFETs with lower threshold voltages and improved subthreshold slope. ${ }^{[47,53,101]}$ This was observed, for example, in blends of TIPS-PEN:PS. ${ }^{[101]}$ By AFM it was shown that smoother and more evenly distributed semiconductor films were found in the blend devices. Also, Raman characterization indicated that blend devices had larger grains, with reduced variation in pentacene backbone orientation along both the channel width and channel length directions than the pure TIPS-PEN devices.

A few studies have recently aimed at unravelling the film depth charge transport by plasma etching blended OSC films in order to shed light on the transport pathways and energy distribution of the localised states with nanometre depth resolution. ${ }^{[102,103]}$ 
Additionally, the use of blends that give rise to bi-layer films allows for the simultaneous deposition of the semiconducting and dielectric layers in a one-step process, demonstrating a facile route for fabricating all-organic transistors. ${ }^{[30,60,92,104,105]}$ PMMA meets the requirements for one-step deposition due to its good dielectric properties, and, furthermore, is a common binder polymer used. In 2008 Qiu et al. reported for the first time the one-step deposition of a P3HT:PMMA blend on $n$-Si(001) in which the PMMA bottom layer of the film was exploited as dielectric. ${ }^{[92]}$ Good performing devices operating at very low voltages were realized following this route. In a similar manner, Marszalek et al. fabricated OFETs based on zone casted films of an $n$-type perylenedicarboximide derivative blended with PMMA. ${ }^{[104]}$

\subsection{Device stability}

OFETs based on OSC:polymer blends typically exhibit an enhanced stability due to several factors such as the improvement of the OSC/dielectric interface quality, the better film crystallinity or the self-encapsulation of the OSC. Kwak et al. reports a blend transistor based on poly(didodecylquaterthiophene-alt-didodecylbithiazole) (PQTBTz-C12) and PS with an electrical performance almost unchanged after three months of environmental exposure. ${ }^{[70]}$ Furthermore, blending $n$-type OSCs with insulating polymer provides a significant stability

improvement. ${ }^{[47-49]}$ In fact, thanks to the use of blends, the OSC DBTTF, which is a non airstable material, could be processed, measured and stored in ambient conditions. ${ }^{[53]}$ The shelfstability of the OFETs was further explored by measuring the devices for several weeks under ambient conditions. The evolution of the mobility and threshold voltage of a set of ten devices measured during 48 days after fabrication could be considered almost constant (Figure 9). 


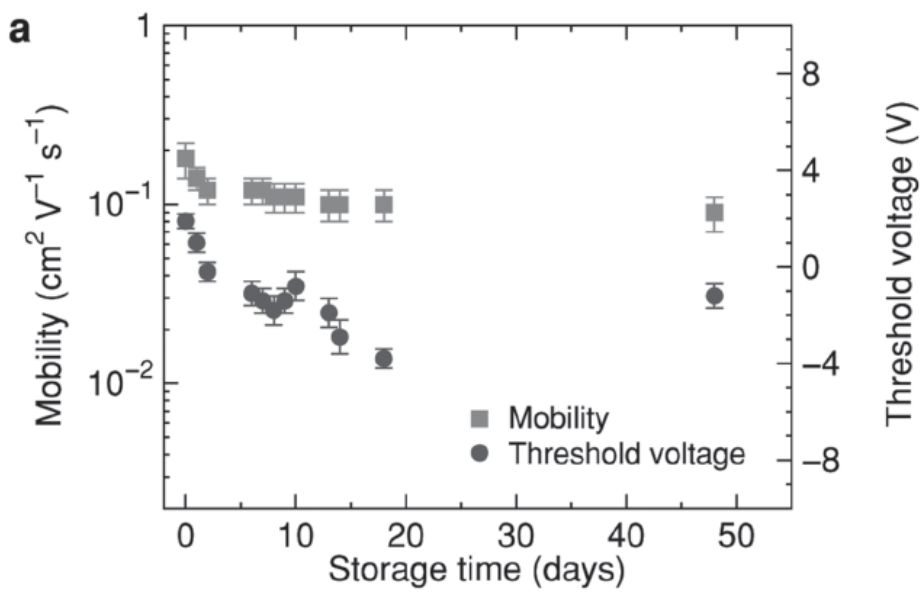

Figure 9. Averaged mobility and threshold voltage values of DBTTF:PS devices measured along 48 days. Reproduced with permission. ${ }^{[53]}$ Copyright 2015, Wiley-VCH.

The OSC:polymer blend layers can also be protected by the formation of a top insulating polymer encapsulation layer, which could act as a barrier against the diffusion of water and/or oxygen. ${ }^{[59]}$ Arias et al. used a blend of the OSC polymer PQT-12 with PMMA that formed a bilayer structure with the self-encapsulated polymer OFETs. The timedependent electrical properties exhibited unchanged electrical properties over exposure to ambient air for 48 hours, meanwhile the subthreshold slope for PQT-12 devices changed from 2.0 V/decade to $4.4 \mathrm{~V} /$ decade after exposure to air for 20 hours; the entire I-V characteristic was shifted, giving a higher switch on voltage and higher off current. As previously mentioned, in some devices this top-encapsulation layer can be as thin as in the order of 1-2 nm, called after skin-layer. ${ }^{[95]}$

Operational stability resulting from applied bias during device operation can also be improved by using OSC:polymer blends. ${ }^{[47,70,106-108]}$ Such improvement is related to the above-mentioned passivation of the substrate by the polymer leading to lower values of density of interface traps. Campos et al. compared the bias stability of OFETs based on PDI8CN2 with and without PS. ${ }^{[4]}$ They found that the $V_{t h}$ shift was much lower in the OFETs based on PDI8CN2:PS than in the ones with neat PDI8CN2 under prolonged bias stress 
(Figure 10). They also estimated the density of states (DoS) distribution of PDI8CN2 and PDI8CN2:PS active layers as indicator of the interface quality. It was described that PDI8CN2:PS films had a sharp distribution of states with up to $\sim 10^{21} \mathrm{~cm}^{-3} \mathrm{eV}^{-1}$ close to the LUMO, in contrast to the more progressive distribution in PDI8CN2 films $\left(\sim 10^{20} \mathrm{~cm}^{-3} \mathrm{eV}^{-1}\right)$ that had a band tail much deeply extended into the band gap.

(a)

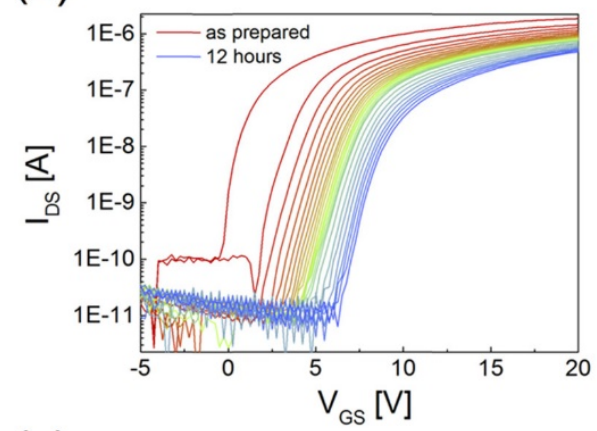

(c)

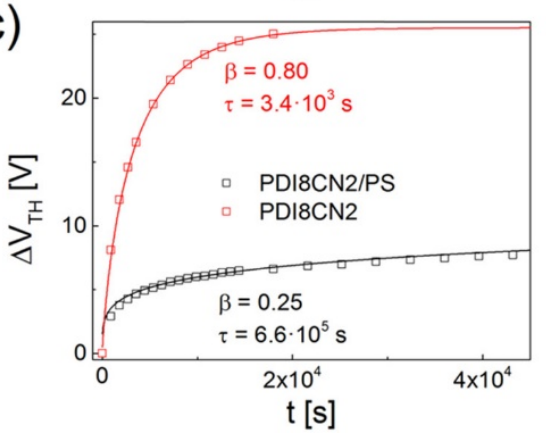

(b)

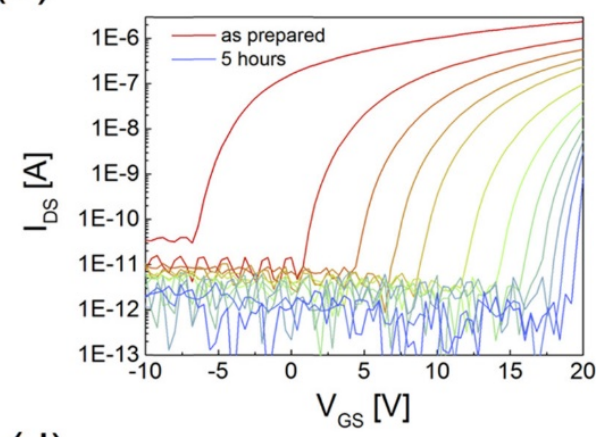

(d)

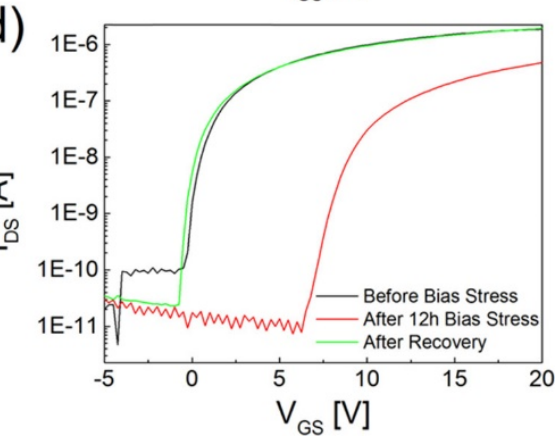

Figure 10. Bias stress stability of (a) PDI8CN2/PS OFET and (b) PDI8CN2 ( $V_{G S}=20 \mathrm{~V}$ and $V_{D S}=0 \mathrm{~V}$ ). (c) $V_{t h}$ shift vs time and fitting curves. (d) Comparison of the transfer characteristics of the sample with PS just before the bias stress measurements, after the bias stress experiments, and after the recovery. Reproduced with permission. ${ }^{[47]}$ Copyright 2018, American Chemical Society.

A high-temperature operation stability might be of great interest for numerous electronic applications. A high performance OFET based on a semicrystalline OSC polymer blended with a polymer matrix with a high glass-transition temperature (higher than the desired working temperature) has been reported to be able to operate at $150{ }^{\circ} \mathrm{C}$ for 6 hours 
(Figure 11). ${ }^{[109]}$ The devices revealed temperature-insensitive charge transport behaviour with hole mobilities stable up to $220{ }^{\circ} \mathrm{C}$. The authors highlighted the importance of the interpenetration of the semiconducting component into the host matrix and the presence of large intermolecular $\pi-\pi$ stacking within semiconducting channels that can be retained at high temperature.
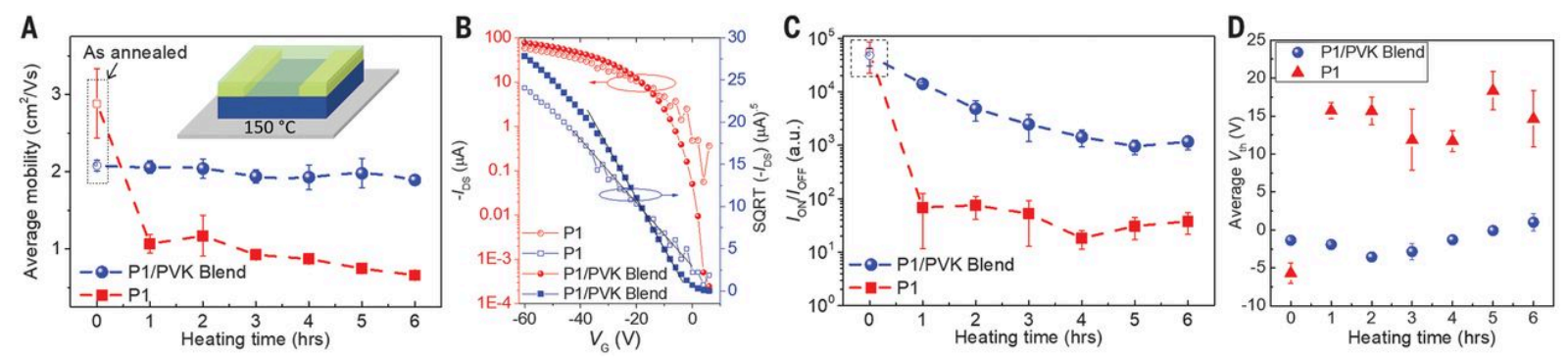

Figure 11. Effect of thermal stress on FET devices and thermal stability. (A) Measured hole mobilities under constant thermal stress for 6 hours. (B) Characteristic transfer curves of FET devices with and without polymer binder (i.e., PVK) after 1 hour of heating. SQRT, square root. (C) Impact of heating on the $I_{O N} / I_{\text {OFF }}$ (D) $V_{\text {th }}$ for FET devices based on blends (below 3 V) and pure OSC (exceeding $20 \mathrm{~V}$ ) upon prolonged heating. Reproduced with permission. ${ }^{[109]}$ Copyright 2018, American Association for the Advancement of Science.

\subsection{Reproducibility}

As mentioned in the first section, the use of blends in the active layer of OFETs permits to reach mobilities in the range of $1-10 \mathrm{~cm}^{2} \mathrm{~V}^{-1} \mathrm{~s}^{-1}$. Moreover, the OSC:polymer blended layers exhibit significantly higher morphology uniformity leading to improved device-to-device reproducibility. ${ }_{[16,34,49,52,53,101,105,108,110]}$ An enhanced reproducibility of thin film morphology, and subsequent thin film transistor performance, depositing a blend of TIPS-PEN with a low permittivity dielectric polymer by inkjet drop ejection process was reported. ${ }^{[110]}$ In addition, Kim et al. also demonstrated that blended devices have considerable 
narrower distribution of mobility. ${ }^{[108]}$ Without the blending polymer, large morphological anisotropy was expected due to the strong interaction between small molecules rather than interaction with the substrate. Importantly for up-scaling to get closer to practical applications, very recently large area reproducibility has been demonstrated in wafer-scale. Flexible OFETs based on a P3HT:PMMA blend were deposited by bar coating with a device yield over $90 \% .^{[105]}$

\subsection{Addition of dopants}

Doping is a key enabler for achieving high-performance OFETs. Several review articles on OFET doping have been recently published showing the high potential of adding dopants in OSCs to control the electrical device characteristics. ${ }^{[111,112]}$ Recently, the electrical performance of blends comprising a small amount of organic semiconductor mixed into an insulating polymer matrix can be improved dramatically upon moderate doping. ${ }^{[113,114]}$ Lu et al. demonstrated the enhanced mobility and on/off ratio of top-contact bottom-gate OFETs based on different polymer p-type OSCs (i.e., P3HT, poly (3-butylthiophene) (P3BT), PQT12 and poly(2,5-bis(3-hexadecylthiophen2-yl)thieno[3,2-b]thiophenes) (PBTTT)) blended with PS or PMMA by depositing on top an ultra-thin layer of the molecular electron acceptor tetrafluoro-tetracyanoquinodimethane (F4-TCNQ) (Figure 12). ${ }^{[113]}$
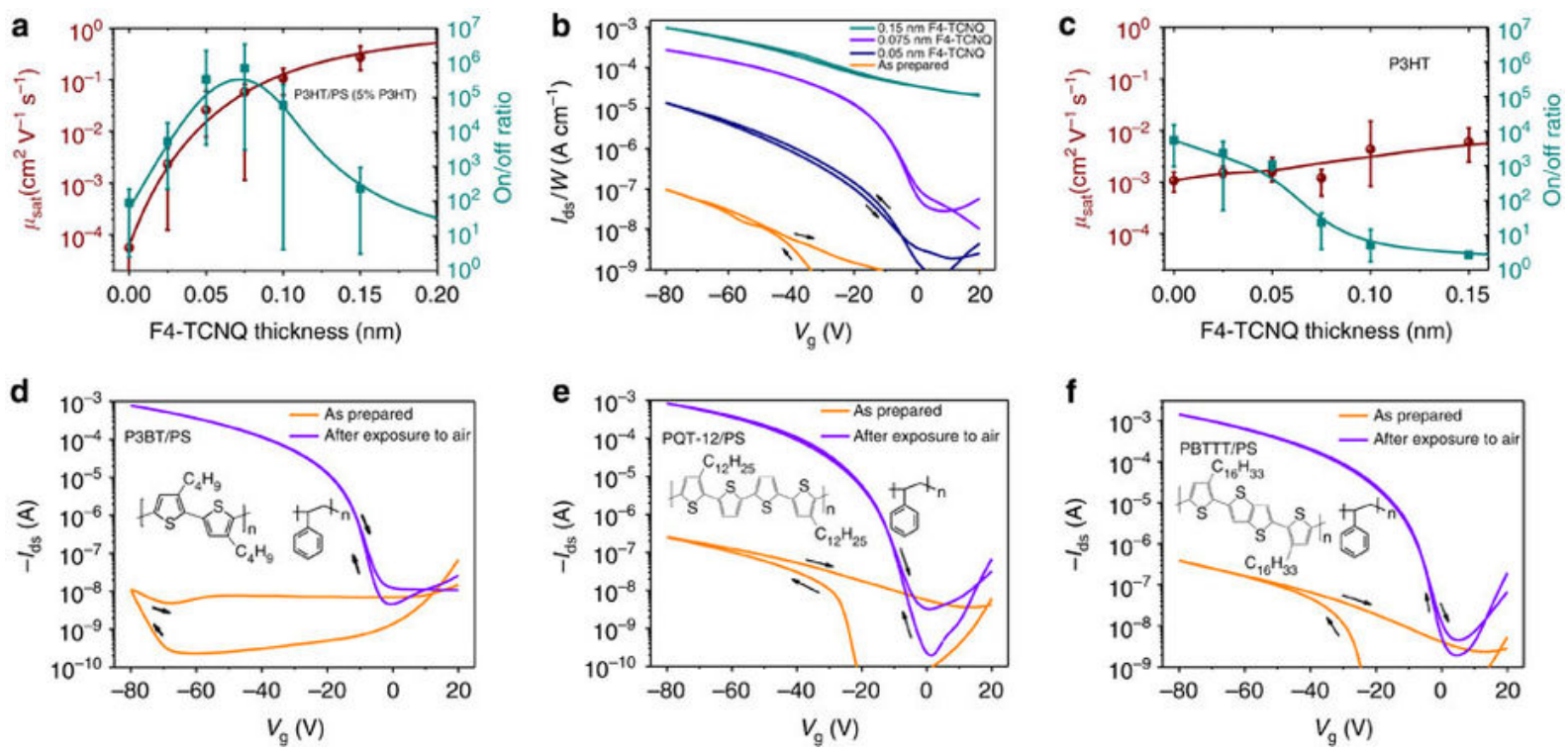
Figure 12. Dependence of mobility and on/off ratio of a P3HT:PS (5\% P3HT) blend transistor on the nominal thickness of F4-TCNQ deposited on top of the blend. Reproduced with permission. ${ }^{[113]}$ Copyright 2013, Springer Nature.

In a parallel work, the p-dopant complex molybdenum tris-[1-(trifluoroethanoyl)-2(trifluoromethyl)ethane1,2-dithiolene] $\left[\mathrm{Mo}\left(\mathrm{tfd}-\mathrm{COCF}_{3}\right)_{3}\right]$ was added directly in the solution of a blend of TIPS-PEN:PS. ${ }^{[114]}$ In the resulting devices the $V_{t h}$ was tuned without affecting the mobility or on/off current ratio.

Despite in this review we focus on organic semiconductors with insulating polymer binders, it worth mentioning the high mobility values that have been obtained by using semiconducting polymers (i.e. poly(dimethyl-triarylamine (PTAA)) as binding polymer. ${ }^{[17,115-}$ ${ }^{117]}$ Recently, admixing the Lewis acid $\mathrm{B}\left(\mathrm{C}_{6} \mathrm{~F}_{5}\right)_{3}$ in the diF-TESADT:PTAA blend has been found to increase the maximum hole mobility of the transistors from $2.5 \mathrm{~cm}^{2} \mathrm{~V}^{-1} \mathrm{~s}^{-1}$ to a record value for this mixture of $>8 \mathrm{~cm}^{2} \mathrm{~V}^{-1} \mathrm{~s}^{-1}$. ${ }^{117]}$ Also, using a blend of C8-BTBT with the binder polymer indacenodithiophene-benzothiadiazole $\left(\mathrm{C}_{16} \mathrm{IDT}-\mathrm{BT}\right)$ doped with the molecule $\mathrm{C}_{60} \mathrm{~F}_{48}$ has shown to improve the overall electrical device performance enhancing the average saturation mobility from 1.4 to $7.8 \mathrm{~cm}^{2} \mathrm{~V}^{-1} \mathrm{~s}^{-1}$. ${ }^{[18]}$ These works clearly illustrate the high potential of adding dopants in organic semiconductor blends and that there are still plenty of open possibilities.

\subsection{Mechanical properties}

Organic semiconductors have been associated with flexible and printed electronics since its inception. In fact, the main characteristic that underpins the most valuable advantages of organic electronic materials is its deformability, especially if they are conceived to be printed by roll-to-roll systems. ${ }^{[119]}$ The fact that OSC:polymer blends are much easier to 
process from solution has therefore open new perspectives towards the development of novel flexible printed devices. ${ }^{[120]}$

The mechanical response of OFETs based on solution-sheared films of TIPS-PEN:PS blends on Parylene C has been investigated as function of the coating direction since it influences the thin film morphology. ${ }^{[121]}$ It was found that devices coated perpendicularly to the channel showed an increase of contact resistance larger than parallel coated ones during elongation, justifying the larger current reduction. In contrast, parallel coated devices showed a larger contact resistance reduction and sensitivity for compression than orthogonal coated ones. In addition, Raghuwanshi et al. reported devices of TIPS-PEN fabricated depositing the OSC on top of a buffer P4VP polymer layer (bi-layer film) or using a blend of the active material with PS and casting it directly on the dielectric (Figure 13). ${ }^{[122,123]}$ Both types of devices exhibited high electrical stability upon bending with increasing magnitude of strain or its duration up to 5 days.

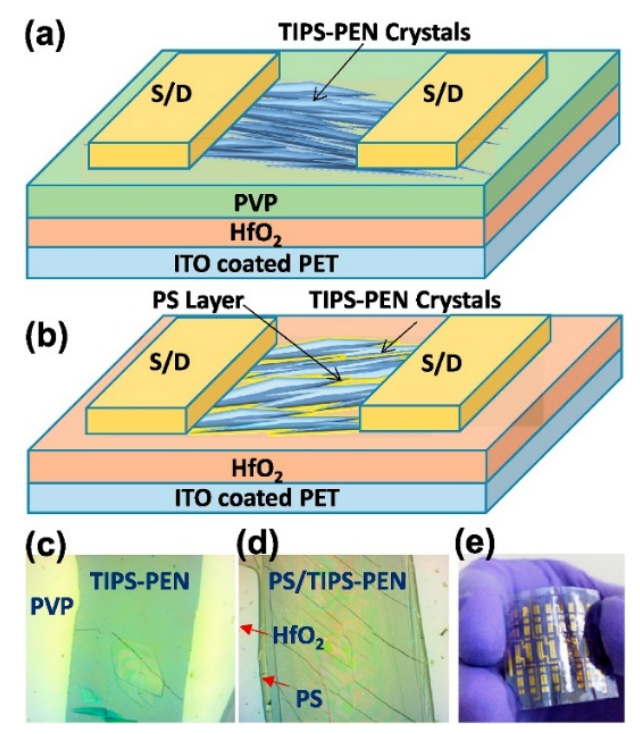

Figure 13. Device structure of flexible top contact (a) TIPS-PEN:PVP bi-layer and (b) TIPSPEN:PS blend OFETs. (c) Optical image of a crystal obtained from the neat TIPS-PEN solution and, (d) TIPS-PEN:PS blend solution, (e) Digital image of flexible TIPS-PEN OFETs. Reproduced with permission. ${ }^{[122]}$ Copyright 2016, Elsevier. 
Important steps towards fully flexible electronics have been recently shown. Very thin bar-coated films prepared from P3HT:PMMA blends to simultaneously fabricate the OSC and dielectric layers were lifted from the substrate to obtain free-standing arrays that could be attached on any surface with varying shapes (Figure 14 a-c). ${ }^{[105]}$ Mechanical properties of elastomers, are appealing in this context due to weak intermolecular forces, low Young's modulus and high failure strain. Highly stretchable polymer semiconductor films have been achieved by the nanoconfinement of nanofibrils polymer semiconductors inside a soft and deformable elastomer in order to improve the stretchability of the polymer OSCs without affecting the charge transport mobility (Figure 14d). ${ }^{[24]}$ Following this approach, the fabricated semiconducting films could be stretched up to $100 \%$ strain without affecting their mobility ( $>1.0 \mathrm{~cm}^{2} \mathrm{~V}^{-1} \mathrm{~s}^{-1}$ ), exceeding their neat counterparts by one to four orders of magnitude.

(a)

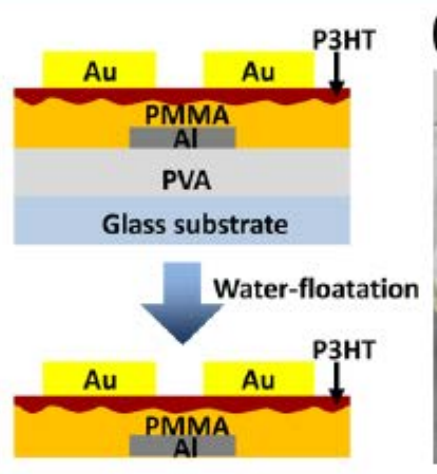

(b)

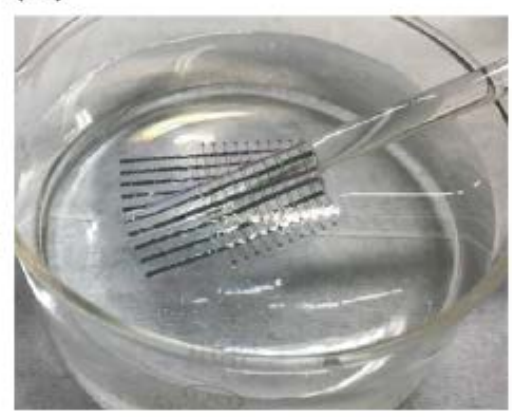

(c)

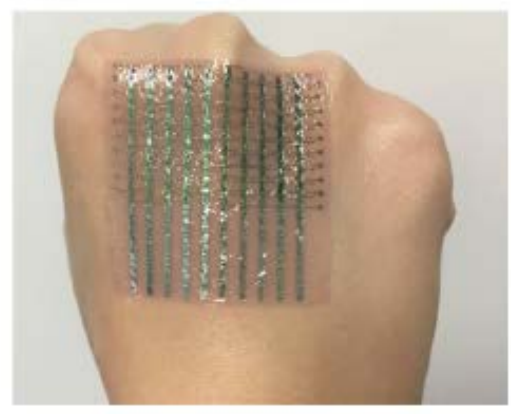

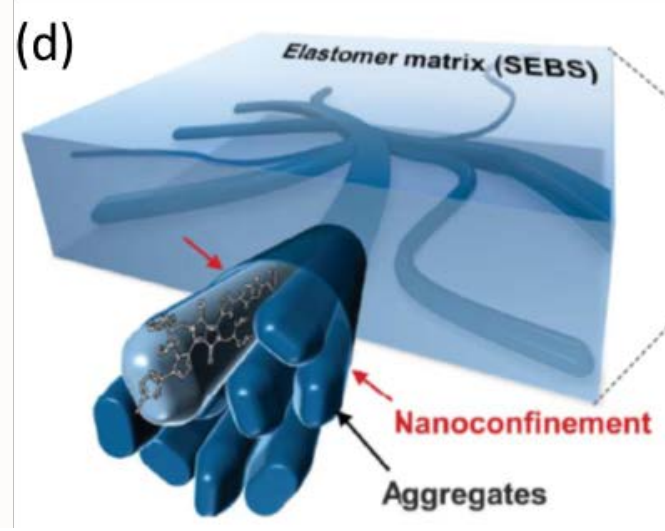

Stretchable

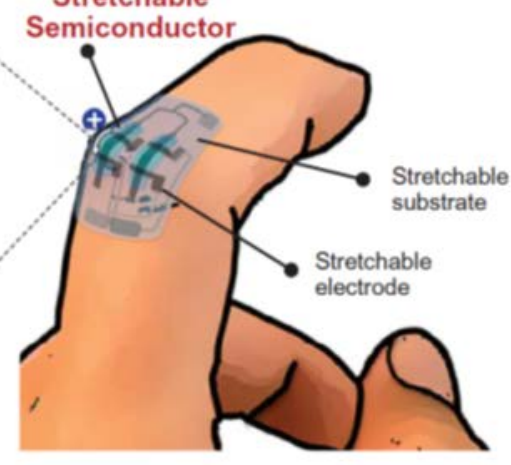

Figure 14 a-c) The device structure and photographic images of free-standing arrays of OFETs based on P3HT:PMMA blends. Reproduced with permission. ${ }^{[105]}$ Copyright 2018, 
American Chemical Society; d) A 3D schematic of the morphology composed of embedded nanoscale networks of polymer semiconductor. Reproduced with permission. ${ }^{[124]}$ Copyright 2017, American Association for the Advancement of Science.

\subsection{Low-voltage OFETs}

Low-voltage OFETs are of great interest for organic electronics applications that require low power consumption. In a field-effect transistor, the gate voltage required to switch the transistor on scales directly with insulator thickness and inversely with insulator dielectric constant. Hence, the use of a polymeric insulator dielectric combined with the blended semiconductors can lead to the fabrication of low-cost and low-voltage OFETs. Recently a ultralow-voltage OFET device (operating at $<1 \mathrm{~V}$ ) has reached a record-high mobility of 4.2 $\mathrm{cm}^{2} \mathrm{~V}^{-1} \mathrm{~s}^{-1}$ for a solution-processed blend of TIPS-PEN:PS deposited on a polymer dielectric layer of polyvinylphenol cross-linked with 4,4'-(hexafluoroisopropylidene)diphthalic anhydride. ${ }^{[29]}$ Previously, low-voltage OFETs using blends as active layer were also reported. ${ }^{[125-127]}$ In 2015, Faraji et al. used a dielectric layer which consists of a bilayer film composed of a high- $k$ nanocomposite film that is partially capped by a low- $k$ polymer. ${ }^{[126]}$ Later on, they reported mobilities in excess of $1 \mathrm{~cm}^{2} \mathrm{~V}^{-1} \mathrm{~s}^{-1}$ at $3 \mathrm{~V}$ using solution-processed cyanoethyl cellulose (CEC) and CEC-based nanocomposites as dielectric layer. ${ }^{[127]}$ These device architectures are suitable for low power electronics applications such as wearable electronics and aqueous biosensors, where low voltage operation is a necessary condition due to the low electrochemical stability window of water.

\section{Organic semiconducting blends applied to electrolyte-gated field-effect transistors}

\section{(EGOFETs)}

One of the most appealing challenges regarding organic-field-effect transistors is represented by the possibility to exploit them as transducers for sensing analytes in aqueous 
media. Thus, a promising OFET transistor configuration for these applications is named Electrolyte-Gated Field-Effect Transistor (EGOFET) or Water-Gated Field-Effect transistors (WGOFET) when pure water is employed as dielectric liquid. In these devices the organic semiconductor is directly exposed to a water medium and a metallic electrode immersed on it acts as gate terminal. The operating mode is based on formation of two electrical double layers (EDLs) at the gate electrode/electrolyte and electrolyte/organic semiconductor interfaces which capacitance can reach values on the order of $\mu \mathrm{F} / \mathrm{cm}^{2}$. The first demonstration of field effect accumulation via water gating was reported in 2010 by Kergoat and coworkers. ${ }^{[128]}$ In this work a P3HT-based organic field effect transistor was biased by different metal gate electrodes through a water medium with a resulting field-effect mobility of $3.9 \mathrm{x}$ $10^{-3} \mathrm{~cm}^{2} \mathrm{~V}^{-1} \mathrm{~s}^{-1}$

Since then the great efforts have been devoted to developing EGOFETs. ${ }^{[129,130]}$ However, for these devices active materials stable in aqueous media are required. As a consequence, the majority of EGOFET thin films have been mainly based on the ultra-high vacuum (UHV) deposition of OSCs giving very crystalline films or on semi-crystalline polymers, like P3HT.

The importance of polymer blending as strategy to improve EGOFET performance was demonstrated one year after they discovery by the same group in a follow-up paper. Here the authors reported an amelioration of a P3HT-based EGOFET by the addition of PMMA to the P3HT OSC solution rising more than one order of magnitude the EGOFET mobility. ${ }^{[131]}$ Nonetheless, it has not been until a few years later that our group has further intensively explored the blending approach in EGOFETs. This has opened the possibility to employ as active materials many different soluble OSCs (i.e., DBTTF, TIPS-Pentacene, diF-TES-ADT, DPTTA, etc.) which, after blending, give devices with an enhanced mobility and stability. ${ }^{[46,132-135]}$ In fact, some of these EGOFET devices based on these formulations can commonly operate in water under continuous electrical stress for more than 10 hours 
displaying an unprecedented robustness. Such extreme stability has been attributed to the high crystallinity of the film and its encapsulation by the polymeric matrix. ${ }^{[53]}$

Furthermore, diF-TES-ADT:PS-based EGOFETs were demonstrated to have a preferred interaction towards mercury cations, a severe environmental pollutant. ${ }^{[136]}$ The simple exposure of the EGOFET to aqueous solutions containing $\mathrm{Hg}^{2+}$ induced a positive shift of the $V_{t h}$ due to the redox reaction taking place between the OSC and the $\mathrm{Hg}^{2+}$ ions, which was selective over other divalent cations (Figure 15). The role of polystyrene was essential to limit the redox reaction on the surface of the OSC, key to realize a sensor. It was found that in the absence of the PS binder, the $\mathrm{Hg}^{2+}$ ions were penetrating into the bulk of the material deteriorating the device. Thus, polymer matrixes can also play a pivotal role in controlling the diffusion of analytes into the active material.
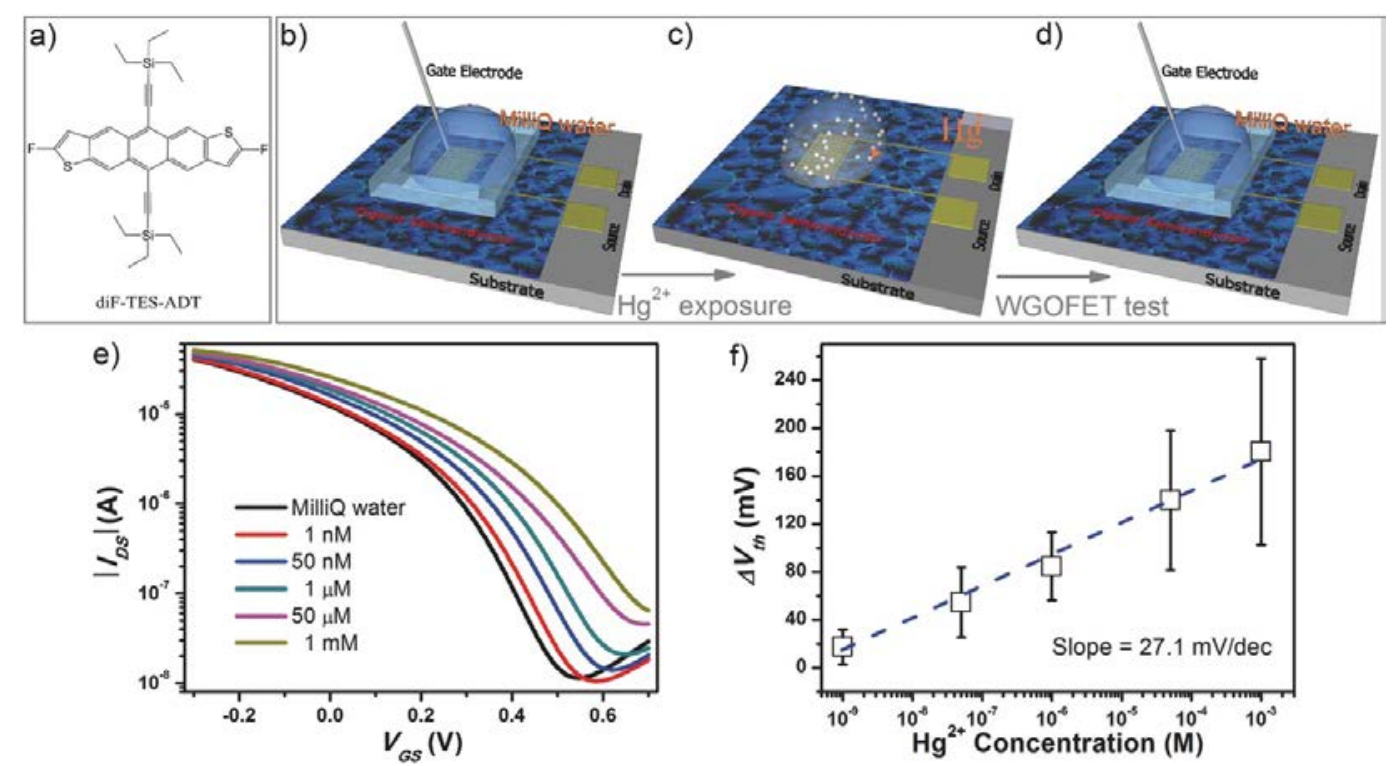

Figure 15. a) Chemical structures of the OSC used as active material for WGOFET. b) WGOFET architecture measured in MilliQ water, c) blend of OSC and binder polymer exposed to $\mathrm{Hg}^{2+}$ solutions, d) WGOFET characterized after $\mathrm{Hg}^{2+}$ exposure. e) Transfer characteristics of WGOFET with different concentration of $\mathrm{Hg}^{2+}$. f) $V_{\text {th }}$ shifts versus $\mathrm{Hg}^{2+}$ 
concentrations. The sample was exposed to $\mathrm{Hg}^{2+}$ from $1 \times 10^{-9}$ to $1 \times 10^{-3} \mathrm{M}$ in ascending order. Reproduced with permission. ${ }^{[136]}$ Copyright 2017, Wiley-VCH.

\section{Future perspectives}

The commercialisation of organic electronics requires more reliable devices with higher stability at low cost production. The utilisation of blends composed by OSCs and insulating binding polymers improves the material processability allowing the deposition of thin films by printing techniques compatible with flexible substrates. In addition, the blends lead to higher reproducibility OFETs and, typically, with an enhanced electrical performance. This is due to an increase in the OSC crystallinity and higher quality of OSC/dielectric interface, which causes a higher operational and environmental stability.

Recent examples reported on devices based on OSC/insulator polymer blends thin films elucidate that this route has significantly step forward towards the development of real applications. For instance, flexible and transparent electronics has been demonstrated ${ }^{[137]}$ as well as printed organic complementary inverters ${ }^{[138]}$ and organic photodiodes with speed enhancement. ${ }^{[139]}$ On the other hand, OSC/insulating polymer blends will also have an impact in the area of conformable wearable electronics that requires stretchable semiconductors $^{[124,140]}$ and, once integrated in EGOFETs, in the field of sensors, including point-of-care applications (PoC) for rapid medical diagnosis reducing health costs in a near future. OFETs have been the most considered device for blends of OSC/binding polymer due to the vertical phase separation of the resulting films. However, OSCs blended in an insulating matrix might have a great interest in other applications such as in thermoelectrics applications because they tend to give higher electrical conductivity maintaining or lowering the thermal conductivity. ${ }^{[141,142]}$

The impressive efforts carried out over the last 30 years to develop organic electronics have resulted in dramatic device improvements but, however, the transition from laboratory to 
the market has lagged behind. The main bottle-necks coupled to lack of reproducibility and stability together with need to develop low cost processing techniques seem to be overcome in a great extent by using blends of OSCs.

\section{Acknowledgements}

The authors thank the ERC StG 2012-306826 e-GAMES project, the Networking Research Center on Bioengineering, Biomaterials and Nanomedicine (CIBER-BBN), the DGI (Spain) project, FANCY CTQ2016-80030-R, the Generalitat de Catalunya (2017-SGR-918) and the Spanish Ministry of Economy and Competitiveness, through the 'Severo Ochoa' Programme for Centres of Excellence in R\&D (SEV- 2015-0496). F. L. and R. P. gratefully acknowledge the "Juan de la Cierva” programme. The authors thank Dr. Xenofon Strakosas for his help with the TOC image.

\section{References}

[1] A. R. Brown, C. P. Jarret, D. M. de Leeuw, M. Matters, Synthetic Metals 1994, 68, 65.

[2] H. Sirringhaus, Advanced Materials 2005, 17, 2411.

[3] M. Mas-Torrent, C. Rovira, Chemical Society Reviews 2008, 37, 827.

[4] S. Allard, M. Forster, B. Souharce, H. Thiem, U. Scherf, Angewandte Chemie International Edition 2008, 47, 4070.

[5] W. Wu, Y. Liu, D. Zhu, Chemical Society Reviews 2010, 39, 1489.

[6] W. Clemens, W. Fix, J. Ficker, A. Knobloch, A. Ullmann, Journal of Materials Research 2004, 19, 1963.

[7] M. Berggren, D. Nilsson, N. D. Robinson, Nature Materials 2007, 6, 3.

[8] J. Perelaer, P. J. Smith, D. Mager, D. Soltman, S. K. Volkman, V. Subramanian, J. G. Korvink, U. S. Schubert, Journal of Materials Chemistry 2010, 20, 8446.

[9] S. Chung, K. Cho, T. Lee, Advanced Science 2019, 1801445.

[10] J. E. Anthony, J. S. Brooks, D. L. Eaton, S. R. Parkin, Journal of the American Chemical Society 2001, 123, 9482.

[11] C. D. Sheraw, T. N. Jackson, D. L. Eaton, J. E. Anthony, Advanced Materials 2003, 15, 2009.

[12] D. Lehnherr, A. R. Waterloo, K. P. Goetz, M. M. Payne, F. Hampel, J. E. Anthony, O. D. Jurchescu, R. R. Tykwinski, Organic Letters 2012, 14, 3660.

[13] J. Smith, R. Hamilton, I. McCulloch, N. Stingelin-Stutzmann, M. Heeney, D. D. C. Bradley, T. D. Anthopoulos, Journal of Materials Chemistry 2010, 20, 2562.

[14] W. H. Lee, Y. D. Park, Polymers 2014, 6, 1057. 
[15] A. D. Scaccabarozzi, N. Stingelin, Journal of Materials Chemistry A 2014, 2, 10818.

[16] S. Riera-Galindo, A. Tamayo, M. Mas-Torrent, ACS Omega 2018, 3, 2329-2339.

[17] A. F. Paterson, S. Singh, K. J. Fallon, T. Hodsden, Y. Han, B. C. Schroeder, H. Bronstein, M. Heeney, I. McCulloch, T. D. Anthopoulos, Advanced Materials 2018, 30, 1801079.

[18] L. J. Richter, D. M. DeLongchamp, A. Amassian, Chemical Reviews 2017, 117, 6332.

[19] G. Horowitz, Advanced Materials 1AD, 10, 365.

[20] N. Stingelin-Stutzmann, E. Smits, H. Wondergem, C. Tanase, P. Blom, P. Smith, D. de Leeuw, Nature Materials 2005, 4, 601.

[21] V. C. Sundar, J. Zaumseil, V. Podzorov, E. Menard, R. L. Willett, T. Someya, M. E. Gershenson, J. A. Rogers, Science 2004, 303, 1644 LP.

[22] P. S. Jo, D. T. Duong, J. Park, R. Sinclair, A. Salleo, Chemistry of Materials 2015, 27, 3979.

[23] W. Tang, L. Feng, P. Yu, J. Zhao, X. Guo, Advanced Electronic Materials 2016, 2, 1500454.

[24] D. K. Hwang, C. Fuentes-Hernandez, J. D. Berrigan, Y. Fang, J. Kim, W. J. Potscavage, H. Cheun, K. H. Sandhage, B. Kippelen, Journal of Materials Chemistry 2012, 22, 5531.

[25] S. Y. Cho, J. M. Ko, J. Lim, J. Y. Lee, C. Lee, Journal of Materials Chemistry C 2013, 1, 914.

[26] B. Park, I.-G. Bae, O. E. Kwon, H. G. Jeon, RSC Advances 2016, 6, 101613.

[27] L.-H. H. Chou, W.-C. C. Chang, G.-Y. Y. He, Y.-C. C. Chiu, C.-L. L. Liu, Reactive and Functional Polymers 2016, 108, 130.

[28] X. Li, W. T. T. Smaal, C. Kjellander, B. Van Der Putten, K. Gualandris, E. C. P. Smits, J. Anthony, D. J. Broer, P. W. M. Blom, J. Genoe, G. Gelinck, Organic Electronics: physics, materials, applications 2011, 12, 1319.

[29] C. Teixeira da Rocha, K. Haase, Y. Zheng, M. Löffler, M. Hambsch, S. C. B. Mannsfeld, Advanced Electronic Materials 2018, $1800141,1$.

[30] W. H. Lee, J. A. Lim, D. Kwak, J. H. Cho, H. S. Lee, H. H. Choi, K. Cho, Advanced Materials 2009, 21, 4243.

[31] R. Hamilton, J. Smith, S. Ogier, M. Heeney, J. E. Anthony, I. McCulloch, J. Veres, D. D. C. C. Bradley, T. D. Anthopoulos, Advanced Materials 2009, 21, 1166.

[32] K. Zhao, O. Wodo, D. Ren, H. U. Khan, M. R. Niazi, H. Hu, M. Abdelsamie, R. Li, E. Q. Li, L. Yu, B. Yan, M. M. Payne, J. Smith, J. E. Anthony, T. D. Anthopoulos, S. T. Thoroddsen, B. Ganapathysubramanian, A. Amassian, Advanced Functional Materials 2016, 26, 1737.

[33] M. R. Niazi, R. Li, E. Qiang Li, A. R. Kirmani, M. Abdelsamie, Q. Wang, W. Pan, M. M. Payne, J. E. Anthony, D.-M. M. Smilgies, S. T. Thoroddsen, E. P. Giannelis, A. Amassian, Nature Communications 2015, 6, 8598.

[34] I. Temiño, F. G. del Pozo, M. R. Ajayakumar, S. Galindo, J. Puigdollers, M. MasTorrent, Advanced Materials Technologies 2016, 1, 1600090.

[35] S. Georgakopoulos, A. Pérez-Rodríguez, A. Campos, I. Temiño, S. Galindo, E. Barrena, C. Ocal, M. Mas-Torrent, Organic Electronics: physics, materials, applications 2017, 48, 365.

[36] K. Haase, C. Teixeira da Rocha, C. Hauenstein, Y. Zheng, M. Hambsch, S. C. B. Mannsfeld, Advanced Electronic Materials 2018, 1800076.

[37] T. Yamamoto, K. Takimiya, Journal of the American Chemical Society 2007, 129, 2224.

[38] U. Zschieschang, F. Ante, D. Kälblein, T. Yamamoto, K. Takimiya, H. Kuwabara, M. Ikeda, T. Sekitani, T. Someya, J. B. Nimoth, H. Klauk, Organic Electronics: physics, materials, applications 2011, 12, 1370. 
[39] K. Kuribara, H. Wang, N. Uchiyama, K. Fukuda, T. Yokota, U. Zschieschang, C. Jaye, D. Fischer, H. Klauk, T. Yamamoto, K. Takimiya, M. Ikeda, H. Kuwabara, T. Sekitani, Y. L. Loo, T. Someya, Nature Communications 2012, 3, 723.

[40] S. Haas, Y. Takahashi, K. Takimiya, T. Hasegawa, Applied Physics Letters 2009, 95, 022111.

[41] W. Xie, K. Willa, Y. Wu, R. Häusermann, K. Takimiya, B. Batlogg, C. D. Frisbie, Advanced Materials 2013, 25, 3478.

[42] Y. Kimura, T. Nagase, T. Kobayashi, A. Hamaguchi, Y. Ikeda, T. Shiro, K. Takimiya, H. Naito, Advanced Materials 2015, 27, 727.

[43] A. Hamaguchi, T. Negishi, Y. Kimura, Y. Ikeda, K. Takimiya, S. Z. Bisri, Y. Iwasa, T. Shiro, Advanced Materials 2015, 27, 6606.

[44] K. Zhang, T. Marszalek, P. Wucher, Z. Wang, L. Veith, H. Lu, H. J. Räder, P. M. Beaujuge, P. W. M. Blom, W. Pisula, Advanced Functional Materials 2018, 28, 1805594.

[45] J. Soeda, T. Okamoto, C. Mitsui, J. Takeya, Organic Electronics: physics, materials, applications 2016, 39, 127.

[46] A. Campos, Q. Zhang, M. R. Ajayakumar, F. Leonardi, M. Mas-Torrent, Advanced Electronic Materials 2017, 1700349.

[47] A. Campos, S. Riera-Galindo, J. Puigdollers, M. Mas-Torrent, ACS Applied Materials and Interfaces 2018, 10, 15952.

[48] H. Zhong, J. Smith, S. Rossbauer, A. J. P. White, T. D. Anthopoulos, M. Heeney, Advanced Materials 2012, 24, 3205.

[49] P. S. K. Amegadze, Y.-Y. Noh, Thin Solid Films 2014, 556, 414.

[50] M. Kang, H. Hwang, W. T. Park, D. Khim, J. S. Yeo, Y. Kim, Y. J. Kim, Y. Y. Noh, D. Y. Kim, ACS Applied Materials and Interfaces 2017, 9, 2686.

[51] D. Ljubic, C. S. Smithson, Y. Wu, S. Zhu, ACS Applied Materials and Interfaces 2016, 8, 3744-3754.

[52] S. Galindo, A. Tamayo, F. Leonardi, M. Mas-Torrent, Advanced Functional Materials 2017, 27, 1700526.

[53] F. G. Del Pozo, S. Fabiano, R. Pfattner, S. Georgakopoulos, S. Galindo, X. Liu, S. Braun, M. Fahlman, J. Veciana, C. Rovira, X. Crispin, M. Berggren, M. Mas-Torrent, Advanced Functional Materials 2016, 26, 2379.

[54] M. R. Niazi, R. Li, M. Abdelsamie, K. Zhao, D. H. Anjum, M. M. Payne, J. Anthony, D.-M. M. Smilgies, A. Amassian, Advanced Functional Materials 2016, 26, 2371.

[55] H. Sirringhaus, P. J. Brown, R. H. Friend, M. M. Nielsen, K. Bechgaard, B. M. W. Langeveld-Voss, A. J. H. Spiering, R. A. J. Janssen, E. W. Meijer, P. Herwig, D. M. de Leeuw, Nature 1999, 401, 685.

[56] E. Mena-Osteritz, A. Meyer, B. M. W. Langeveld-Voss, R. A. J. Janssen, E. W. Meijer, P. Bäuerle, Angewandte Chemie International Edition 2000, 39, 2679.

[57] S. Goffri, C. Müller, N. Stingelin-Stutzmann, D. W. Breiby, C. P. Radano, J. W. Andreasen, R. Thompson, R. A. J. Janssen, M. M. Nielsen, P. Smith, H. Sirringhaus, Nature Materials 2006, 5, 950.

[58] L. Qiu, X. Wang, W. H. Lee, J. A. Lim, J. S. Kim, D. Kwak, K. Cho, Chemistry of Materials 2009, 21, 4380.

[59] A. C. Arias, F. Endicott, R. A. Street, Advanced Materials 2006, 18, 2900.

[60] X. X. Wang, W. H. Lee, G. Zhang, X. X. Wang, B. Kang, H. Lu, L. Qiu, K. Cho, Journal of Materials Chemistry C 2013, 1, 3989.

[61] C. Cho, S. C. C. Kuo, Y. C. S. Chang, Polymer Journal 2018, 50, 975.

[62] Y. Lei, P. Deng, M. Lin, X. Zheng, F. Zhu, B. S. Ong, Advanced Materials 2016, 6687.

[63] L. Qiu, Q. Xu, W. H. Lee, X. Wang, B. Kang, G. Lv, K. Cho, Journal of Materials Chemistry 2011, 21, 15637. 
[64] H.-J. Yun, G. B. Lee, D. S. Chung, Y.-H. Kim, S.-K. Kwon, Advanced Materials 2014, 26, 6612.

[65] M. J. Ford, M. Wang, S. N. Patel, H. Phan, R. A. Segalman, T. Q. Nguyen, G. C. Bazan, Chemistry of Materials 2016, 28, 1256.

[66] L. Tang, P. He, X. Qiao, Q. Qian, H. Li, Mater. Chem. Front. 2017, 1, 2265.

[67] L. Bu, M. Hu, W. Lu, Z. Wang, G. Lu, Advanced Materials 2018, 30, 1704695.

[68] G. Zhang, H. Yang, L. He, L. Hu, S. Lan, F. Li, H. Chen, T. Guo, Journal of Polymer Science Part B: Polymer Physics 2016, 54, 1760.

[69] H. Yang, G. Zhang, J. Zhu, W. He, S. Lan, L. Liao, H. Chen, T. Guo, Journal of Physical Chemistry C 2016, 120, 17282.

[70] D. Kwak, H. H. Choi, B. Kang, D. H. Kim, W. H. Lee, K. Cho, Advanced Functional Materials 2016, 26, 3003.

[71] J. H. Lee, Y. H. Lee, Y. H. Ha, J. Kwon, S. Pyo, Y.-H. Kim, W. H. Lee, RSC Advances 2017, 7, 7526.

[72] A. A. Virkar, S. Mannsfeld, Z. Bao, N. Stingelin, Advanced Materials 2010, 22, 3857.

[73] M. Chang, D. Choi, G. Wang, N. Kleinhenz, N. Persson, B. Park, E. Reichmanis, ACS Applied Materials and Interfaces 2015, 7, 14095-14103.

[74] S. Wang, S. Fabiano, S. Himmelberger, S. Puzinas, X. Crispin, A. Salleo, M. Berggren, Proceedings of the National Academy of Sciences 2015, 112, 10599.

[75] A. Kadashchuk, F. Tong, R. Janneck, I. I. Fishchuk, A. Mityashin, E. Pavlica, A. Köhler, P. Heremans, C. Rolin, G. Bratina, J. Genoe, Physical Review B 2017, 96, 125202.

[76] C. E. Murphy, L. Yang, S. Ray, L. Yu, S. Knox, N. Stingelin, Journal of Applied Physics 2011, 110, 093523.

[77] A. B. Naden, J. Loos, D. A. MacLaren, Journal of Materials Chemistry C 2014, 2, 245.

[78] D. J. Gundlach, J. E. Royer, S. K. Park, S. Subramanian, O. D. Jurchescu, B. H. Hamadani, A. J. Moad, R. J. Kline, L. C. Teague, O. Kirillov, C. A. Richter, J. G. Kushmerick, L. J. Richter, S. R. Parkin, T. N. Jackson, J. E. Anthony, Nature Materials 2008, 7, 216.

[79] R. Li, J. W. Ward, D. M. Smilgies, M. M. Payne, J. E. Anthony, O. D. Jurchescu, A. Amassian, Advanced Materials 2012, 24, 5553.

[80] N. Shin, J. Kang, L. J. Richter, V. M. Prabhu, R. J. Kline, D. A. Fischer, D. M. Delongchamp, M. F. Toney, S. K. Satija, D. J. Gundlach, B. Purushothaman, J. E. Anthony, D. Y. Yoon, Advanced Functional Materials 2013, 23, 366.

[81] Z. He, D. Li, D. K. Hensley, A. J. Rondinone, J. Chen, Applied Physics Letters 2013, 103, 113301.

[82] M. Lada, M. J. Starink, M. Carrasco, L. Chen, P. Miskiewicz, P. Brookes, M. Obarowska, D. C. Smith, Journal of Materials Chemistry 2011, 21, 11232.

[83] J. H. Lee, H. H. Choi, Y. D. Park, J. E. Anthony, J. A. Lim, J. Cho, D. S. Chung, J. Hwang, H. W. Jang, K. Cho, W. H. Lee, L. J. Hun, C. H. Ho, P. Y. Don, A. J. E., L. J. Ah, C. Jangwhan, C. D. Sung, H. Jinhyun, J. H. Won, C. Kilwon, L. W. Hyoung, Advanced Functional Materials 2018, 28, 1802875.

[84] J. H. Lee, Y. Seo, Y. D. Park, J. E. Anthony, D. H. Kwak, J. A. Lim, S. Ko, H. W. Jang, K. Cho, W. H. Lee, Scientific Reports 2019, 9, 21.

[85] J. Cornil, S. Verlaak, N. Martinelli, A. Mityashin, Y. Olivier, T. Van Regemorter, G. D’Avino, L. Muccioli, C. Zannoni, F. Castet, D. Beljonne, P. Heremans, Accounts of Chemical Research 2013, 46, 434.

[86] J. Hoon Park, H. Lim, H. Cheong, K. Min Lee, H. Chul Sohn, G. Lee, S. Im, Organic Electronics: physics, materials, applications 2012, 13, 1250.

[87] A. Shehu, S. D. Quiroga, P. D’Angelo, C. Albonetti, F. Borgatti, M. Murgia, A. Scorzoni, P. Stoliar, F. Biscarini, Physical Review Letters 2010, 104, 1. 
[88] N. Onojima, T. Ozawa, T. Sugai, S. Obata, Y. Miyazawa, J. Yamanaka, Organic Electronics: physics, materials, applications 2019, 66, 206.

[89] T. Ohe, M. Kuribayashi, R. Yasuda, A. Tsuboi, K. Nomoto, K. Satori, M. Itabashi, J. Kasahara, Applied Physics Letters 2008, 93, 053303.

[90] J. Kang, N. Shin, Y. J. Do, V. M. Prabhu, D. Y. Yoon, Journal of the American Chemical Society 2008, 130, 12273.

[91] M.-B. Madec, D. Crouch, G. R. Llorente, T. J. Whittle, M. Geoghegan, S. G. Yeates, Journal of Materials Chemistry 2008, 18, 3230.

[92] L. Qiu, J. A. Lim, X. Wang, W. H. Lee, M. Hwang, K. Cho, Advanced Materials 2008, 20, 1141.

[93] M. Kang, H. Hansu, W.-T. Park, D. Khim, J.-S. Yeo, Y. Kim, Y.-J. Kim, Y.-Y. Noh, D.-Y. Kim, ACS Applied Materials and Interfaces 2017, 9, 2686.

[94] W. H. Lee, D. Kwak, J. E. Anthony, H. S. Lee, H. H. Choi, D. H. Kim, S. G. Lee, K. Cho, Advanced Functional Materials 2012, 22, 267.

[95] A. Pérez-Rodríguez, I. Temiño, C. Ocal, M. Mas-Torrent, E. Barrena, ACS Applied Materials \& Interfaces 2018, 10, 7296-7303.

[96] M. Mas-Torrent, C. Rovira, Chemical Reviews 2011, 111, 4833.

[97] R. Pfattner, S. T. Bromley, C. Rovira, M. Mas-Torrent, Advanced Functional Materials 2016, 26, 2256.

[98] Y. Diao, K. M. Lenn, W.-Y. Lee, M. A. Blood-Forsythe, J. Xu, Y. Mao, Y. Kim, J. A. Reinspach, S. Park, A. Aspuru-Guzik, G. Xue, P. Clancy, Z. Bao, S. C. B. Mannsfeld, Journal of American Chemical Society 2014, 136, 17046-17057.

[99] M. H. Yoon, C. Kim, A. Facchetti, T. J. Marks, Journal of the American Chemical Society 2006, 128, 12851.

[100] F. C. Chen, C. H. Liao, Applied Physics Letters 2008, 93, 2006.

[101] D. T. James, B. K. C. Kjellander, W. T. T. Smaal, G. H. Gelinck, C. Combe, I. McCulloch, R. Wilson, J. H. Burroughes, D. D. C. Bradley, J. S. Kim, ACS nano 2011, 5, 9824.

[102] L. Bu, S. Gao, W. Wang, L. Zhou, S. Feng, X. Chen, D. Yu, S. Li, G. Lu, Advanced Electronic Materials 2016, 2, 1600359.

[103] G. Lu, R. Di Pietro, L. S. Kölln, I. Nasrallah, L. Zhou, S. Mollinger, S. Himmelberger, N. Koch, A. Salleo, D. Neher, Advanced Electronic Materials 2016, 2, 1600267.

[104] T. Marszalek, M. Wiatrowski, E. Dobruchowska, J. Jung, J. Ulanski, Journal of Materials Chemistry C 2013, 1, 3190.

[105] F. Ge, Z. Liu, S. B. Lee, X. Wang, G. Zhang, H. Lu, K. Cho, L. Qiu, ACS Applied Materials and Interfaces 2018, 10, 21510.

[106] J. Lee, J. Young Jung, D. Hwan Kim, J. Y. Kim, B. L. Lee, J. Il Park, J. Won Chung, J. Seok Park, B. Koo, Y. Wan Jin, S. Lee, Applied Physics Letters 2012, 100, 51.

[107] D. Bharti, S. P. Tiwari, Synthetic Metals 2016, 221, 186.

[108] Y. H. Kim, J. E. Anthony, S. K. Park, Organic Electronics: physics, materials, applications 2012, 13, 1152.

[109] A. Gumyusenge, D. T. Tran, X. Luo, G. M. Pitch, Y. Zhao, K. A. Jenkins, T. J. Dunn, A. L. Ayzner, B. M. Savoie, J. Mei, Science 2018, 362, 1131.

[110] M.-B. Madec, P. J. Smith, A. Malandraki, N. Wang, J. G. Korvink, S. G. Yeates, Journal of Materials Chemistry 2010, 20, 9155.

[111] I. E. Jacobs, A. J. Moulé, Advanced Materials 2017, 29, 1703063.

[112] Y. Xu, H. Sun, A. Liu, H.-H. Zhu, W. Li, Y.-F. Lin, Y.-Y. Noh, Advanced Materials 2018, 30, 1801830.

[113] G. Lu, J. Blakesley, S. Himmelberger, P. Pingel, J. Frisch, I. Lieberwirth, I. Salzmann, M. Oehzelt, R. Di Pietro, A. Salleo, N. Koch, D. Neher, Nature communications 2013, 4, 1588. 
[114] J. Belasco, S. K. Mohapatra, Y. Zhang, S. Barlow, S. R. Marder, A. Kahn, Applied Physics Letters 2014, 105, 063301.

[115] J. Smith, W. Zhang, R. Sougrat, K. Zhao, R. Li, D. Cha, A. Amassian, M. Heeney, I. McCulloch, T. D. Anthopoulos, Advanced Materials 2012, 24, 2441.

[116] A. F. Paterson, N. D. Treat, W. Zhang, Z. Fei, G. Wyatt-Moon, H. Faber, G. Vourlias, P. A. Patsalas, O. Solomeshch, N. Tessler, M. Heeney, T. D. Anthopoulos, Advanced Materials 2016, 28, 7791.

[117] J. Panidi, A. F. Paterson, D. Khim, Z. Fei, Y. Han, L. Tsetseris, G. Vourlias, P. A. Patsalas, M. Heeney, T. D. Anthopoulos, Advanced Science 2017, 5, 1700290.

[118] A. F. Paterson, Y. H. Lin, A. D. Mottram, Z. Fei, M. R. Niazi, A. R. Kirmani, A. Amassian, O. Solomeshch, N. Tessler, M. Heeney, T. D. Anthopoulos, Advanced Electronic Materials 2018, 4, 1700464.

[119] S. E. Root, S. Savagatrup, A. D. Printz, D. Rodriquez, D. J. Lipomi, Chemical Reviews 2017, 117, 6467.

[120] S. Georgakopoulos, F. G. Del Pozo, M. Mas-Torrent, Journal of Materials Chemistry C 2015, 3, 12199.

[121] S. Lai, I. Temiño, T. Cramer, F. G. del Pozo, B. Fraboni, P. Cosseddu, A. Bonfiglio, M. Mas-Torrent, Advanced Electronic Materials 2017, 1700271.

[122] V. Raghuwanshi, D. Bharti, I. Varun, A. K. Mahato, S. P. Tiwari, Organic Electronics: physics, materials, applications 2016, 34, 284.

[123] D. Bharti, V. Raghuwanshi, I. Varun, A. Kumar Mahato, S. Prakash Tiwari, S. Member, IEEE Electron Device Letters 2016, 37, 1215.

[124] J. Xu, S. Wang, G. N. Wang, C. Zhu, S. Luo, L. Jin, X. Gu, S. Chen, V. R. Feig, J. W. F. To, S. Rondeau-gagné, J. Park, B. C. Schroeder, C. Lu, J. Y. Oh, Y. Wang, Y. Kim, H. Yan, R. Sinclair, D. Zhou, G. Xue, B. Murmann, C. Linder, W. Cai, J. B. Tok, Science 2017, 355, 59.

[125] L. Feng, J. Zhao, W. Tang, X. Xu, X. Guo, Journal of Display Technology 2014, 10, 971.

[126] S. Faraji, T. Hashimoto, M. L. Turner, L. A. Majewski, Organic Electronics: physics, materials, applications 2015, 17, 178.

[127] S. Faraji, E. Danesh, D. J. Tate, M. L. Turner, L. A. Majewski, Journal of Physics D: Applied Physics 2016, 49, 185102.

[128] L. Kergoat, L. Herlogsson, D. Braga, B. Piro, M.-C. Pham, X. Crispin, M. Berggren, G. Horowitz, Advanced Materials 2010, 22, 2565.

[129] E. Macchia, K. Manoli, B. Holzer, C. Di Franco, M. Ghittorelli, F. Torricelli, D. Alberga, G. F. Mangiatordi, G. Palazzo, G. Scamarcio, L. Torsi, Nature Communications 2018, 9, 3223.

[130] M. Berto, C. Diacci, R. D’Agata, M. Pinti, E. Bianchini, M. Di Lauro, S. Casalini, A. Cossarizza, M. Berggren, D. Simon, G. Spoto, F. Biscarini, C. A. Bortolotti, Advanced Biosystems 2018, 2, 1700072.

[131] L. Kergoat, N. Battaglini, L. Miozzo, B. Piro, M.-C. Pham, A. Yassar, G. Horowitz, Organic Electronics: physics, materials, applications 2011, 12, 1253.

[132] F. Leonardi, S. Casalini, Q. Zhang, S. Galindo, D. Gutiérrez, M. Mas-Torrent, Advanced Materials 2016, 28, 10311.

[133] Q. Zhang, F. Leonardi, S. Casalini, I. Temiño, M. Mas-Torrent, Scientific Reports 2016, 6, 39623.

[134] F. Leonardi, A. Tamayo, S. Casalini, M. Mas-Torrent, RSC Advances 2018, 8, 27509.

[135] F. Leonardi, Q. Zhang, Y.-H. Kim, M. Mas-Torrent, Materials Science in Semiconductor Processing 2019, 93, 105.

[136] Q. Zhang, F. Leonardi, S. Casalini, M. Mas-Torrent, Advanced Functional Materials 2017, 27, 1703899. 
[137] K. Yu, B. Park, G. Kim, C.-H. Kim, S. Park, J. Kim, S. Jung, S. Jeong, S. Kwon, H. Kang, J. Kim, M.-H. Yoon, K. Lee, Proceedings of the National Academy of Sciences 2016, 113, 14261.

[138] Y. Takeda, Y. Yoshimura, R. Shiwaku, K. Hayasaka, T. Sekine, T. Okamoto, H. Matsui, D. Kumaki, Y. Katayama, S. Tokito, Advanced Electronic Materials 2018, 4, 1700313.

[139] N. Strobel, R. Eckstein, J. Lehr, U. Lemmer, G. Hernandez-Sosa, Advanced Electronic Materials 2017, 1700345.

[140] S. Wang, J. Xu, W. Wang, G.-J. N. Wang, R. Rastak, F. Molina-Lopez, J. W. Chung, S. Niu, V. R. Feig, J. Lopez, T. Lei, S.-K. Kwon, Y. Kim, A. M. Foudeh, A. Ehrlich, A. Gasperini, Y. Yun, B. Murmann, J. B.-H. Tok, Z. Bao, Nature 2018, 555, 83.

[141] G. Lu, L. Bu, S. Li, X. Yang, Advanced Materials 2014, 26, 2359.

[142] D. Kiefer, L. Yu, E. Fransson, A. Gómez, D. Primetzhofer, A. Amassian, M. CampoyQuiles, C. Müller, Advanced Science 2017, 4, 1600203. 